\title{
III. Die Reaktion in der ethischen Theoriebildung des Theologen
}

Während Karl Bonhoeffer als ärztlicher Gutachter mit der Zwangssterilisation praktisch konfrontiert wurde, erfolgte bei seinem Sohn Dietrich Bonhoeffer die Auseinandersetzung im Modus der theologisch-ethischen Theoriebildung. $\mathrm{Zu}$ gleich konkretisierte sich das Problem aber auch in der kirchlichen Auseinandersetzung um die „Euthanasie“-Frage. Dietrich Bonhoeffers spezielles Interesse an diesen Fragen im Grenzbereich zwischen Medizin und Ethik kam in seiner Beteiligung an der kirchlichen Eugenik-Diskussion und nachfolgend im ethischen Entwurf zum Ausdruck.

\section{Dietrich Bonhoeffers Engagement in der Diskussion um „Rassenfragen“ und sein Einsatz gegen die „Euthanasie“}

Schon in den zwanziger Jahren hatte Dietrich Bonhoeffer mehrfach an Treffen der „Fachkonferenz für Mediziner und Theologen“, einem von der Apologetischen Centrale in Berlin-Spandau organisierten, jährlich stattfindenden interdisziplinären Treffen, teilgenommen. Eugenik war dabei Thema mehrerer Sitzungen ${ }^{1}$. Im Mai 1933 wurde er als Mitglied eines kirchlichen Fachkreises für „Rassenfragen (Eugenik)" in Aussicht genommen. Aufgabe dieses Kreises sollte es sein, eine spätere offizielle „Stellungnahme zur gegenwärtigen Lage“ als Äußerung der vorläufigen Kirchenführung auf „brennende Zeitfragen“ vorzubereiten und in eine sachliche „Aussprache und Auseinandersetzung mit den Vertretern der Glaubensbewegung ,Deutsche Christen “ einzutreten². Daß es zu der geplanten Stellungnahme nie gekommen ist, ist den sich überstürzenden Ereignis-

${ }^{1}$ Vgl. die Übersicht bei Müller-Braunschweig, Berliner Arbeitsgemeinschaft. Die Protokolle wurden 1937 von der Gestapo beschlagnahmt, 1945 von der Sowjetunion kassiert, 1959 in das Deutsche Zentralarchiv in Potsdam überführt, 1991 dem Archiv des Diakonischen Werkes übergeben. In dem nunmehr unvollständigen Bestand fehlen die Protokolle zu den hier interessierenden Sitzungen. Nicht eingesehen werden konnte ein immer noch in Moskau lagernder Restbestand; vgl. Jena/Lenz, Deutsche Bestände.

2 Als Mitglieder waren neben Bonhoeffer vorgesehen Schreiner, Verschuer, Althaus, Künneth, später auch Weichert und „speziell für die Behandlung der eugenetischen Fragen “ Harmsen; vgl. ADW, AC 26 Jungreformatorische Bewegung, Bl. $89 \mathrm{ff}$., Aufzeichnungen Künneth; EZA, 1 A 4/6 Vorschlagsliste, 5.5. 1933; ebenda, Hosemann an Marahrens/Hesse, 6.5. 1933; ebenda, Hosemann an Fachgruppenleiter, 6.5. 1933; LKA Hannover, L 2 Nr. 4 a Bd. II masch. Bespr. im KBA; HA Bethel 2/39-176, Bl. 3-5, Bodelschwingh an Braune, 6.5. 1933. - Einen Teil dieser Belege verdanke ich Dr. Herbert Anzinger. 
sen des Kirchenkampfes zuzuschreiben: Am 27. Mai wurde von Bodelschwingh zum Reichsbischof gewählt, am 6. Juni trat Kapler zurück, am 24.Juni von Bodelschwingh ${ }^{3}$.

An der ersten Synode der Bekennenden Kirche, auf der die „Euthanasie“ diskutiert wurde (9. Synode der Altpreußischen Union im Oktober 1940), konnte Bonhoeffer nicht teilnehmen, wurde jedoch unmittelbar danach vom Bruderrat aufgefordert, „über bestimmte theologische Themen zu arbeiten und das Ergebnis dem preußischen Bruderrat vorzulegen “4. Inwiefern damit eventuell auch eine theologische Arbeit über die in diesen Tagen im Bruderrat intensiv diskutierte "Euthanasie“-Aktion gemeint war", kann den unklar gehaltenen und gerade zur „Euthanasie“-Aktion aus Sicherheitsgründen extrem unvollständig überlieferten Protokollen nicht entnommen werden ${ }^{6}$.

Bonhoeffer stand mit von Bodelschwingh in Kontakt, wußte genau um die drohende „Euthanasie"-Aktion in Bethel" und wurde auch von dem württembergischen Bischof Wurm in dessen Streiten gegen die „Euthanasie“ um ein theologisches Gutachten zugezogen ${ }^{8}$. Während einer Reise in die Schweiz (24.2.-24.3. 1941) hatte er den Generalsekretär des sich formierenden Weltkirchenrates, Willem Visser't Hooft, von der „Euthanasie“-Aktion in Kenntnis gesetzt: „Euthanasie geschieht weiter. Niemand weiß, wieviel Menschen getötet worden sind, aber man nimmt allgemein an, daß die Zahl sehr hoch ist. Es hat eine Reihe von tapferen, aber unwirksamen Protesten gegeben, so durch Bischof Wurm und Kardinal Faulhaber und durch einige Ärzte. Bodelschwingh hat sich geweigert, die Fragebogen für die Selektion auszufüllen und so sind bisher in Bethel keine Patienten getötet worden " ${ }^{\text {'9 }}$. Visser't Hooft gab die Informationen u. a. an George Bell, den Lordbischof von Chichester, weiter, der seinerseits auf diese Nachrichten 1941 in einer über BBC gesendeten „Weihnachtsbotschaft an die Christen in Deutschland" reagierte, in der unter anderem die christlichen Gemeinden zum Widerstand gegen die „Euthanasie“ ermutigt wurden ${ }^{10}$. Möglicherweise ist auch der Informant, der im Herbst 1940 detaillierte Informationen über die Bedrohung Bethels durch die

${ }^{3}$ Vgl. Scholder, Kirchen I, S. 442, 449.

${ }^{4}$ EZA, 50615, Bl. 369, Beschluß des Bruderrates, 26.11. 1940; Bethge, Bonhoeffer, S. 784.

${ }^{5}$ So beschloß der Bruderrat laut Protokoll vom 12.11. 1940, es solle ein "Bruder ${ }^{*}$ (Name nicht genannt) beauftragt werden, „über die Haltung des Centralausschusses zur Frage der Ausmerzung des gemeinschaftsunfähigen Lebens Klarheit zu erlangen", am 26.11. 1940, man möge „einen Brief" (Inhalt nicht genannt) an den Reichsjustizminister senden und Pfarrer Ernst Wilm beauftragen, "mit Fritz von Bodelschwingh über dessen Stellungnahme zur Euthanasie zu sprechen“; EZA, 50615, Bl. 328, 330, 363.

- Auffällig erscheint, daß aus den gleichzeitig zur Frauenordination tagenden Ausschüssen reichhaltig Material überliefert ist; vgl. Globig, Frauenordination, S. 49-68.

7 Siehe S. $104 \mathrm{f}$.

${ }^{8}$ Wir wissen davon nur aus Briefen Bonhoeffers an Bethge; vgl. GS II, S. 404f., 17.2. 1941, 19.2. 1941. Karl Bonhoeffer hatte in dieser Zeit mit Bischof Wurm Kontakt, siehe S. $106 \mathrm{f}$.

9 So ein "confidential report" vom März 1941, den Visser't Hooft für englische Kollegen anfertigte; vgl. GS VI, S. $621 \mathrm{f}$.

${ }^{10} \mathrm{Vgl}$. Bell, Kirche, S.10ff. 
„Euthanasie“-Aktion an den amerikanischen Journalisten William Shirer weitergab $^{11}$, in Bonhoeffers Umfeld zu suchen; einige Indizien sprechen dafür ${ }^{12}$.

In einem Schreiben vom November 1941, das den Generälen Keitel und Falkenhausen zugeleitet werden sollte, ermutigten Dietrich Bonhoeffer und sein Freund Friedrich Justus Perels die Generäle zum Widerstand gegen das Regime und wiesen zur Begründung auch auf die „Euthanasie“-Aktion hin: „Die Tötung sogenannten unwerten Lebens, die nun breiter in den Gemeinden bekannt geworden ist und ihre Opfer gefunden hat, wird von den Christen aller Konfessionen im Zusammenhang mit der allgemeinen Auflösung der zehn Gebote und jeder Rechtssicherheit gesehen und damit als Zeichen der antichristlichen Haltung leitender Stellen mit tiefster Beunruhigung und mit Abscheu aufgenommen "13. Bethge vermutet, daß der Inhalt dieses Schreibens möglicherweise auch Gegenstand der 10. Synode der Bekennenden Kirche der Altpreußischen Union im November 1941 in Hamburg war, an der Dietrich Bonhoeffer wegen einer Lungenentzündung abermals nicht teilnehmen konnte ${ }^{14}$. Nach den ersten Juden-Deportationen berief der Bruderrat der Bekennenden Kirche der Altpreußischen Union Bonhoeffer in einen Ausschuß, der sich in seiner ersten Sitzung am 10. August 1942 mit einem „Gutachten zur Judenfrage, zur Tötung der Geisteskranken, zur Kirchenzucht“ befaßte ${ }^{15}$. Wiederum fehlen Gut-

11 Vgl. Shirer, Berlin Diary, S.446f., 449f. Shirer veröffentlichte sein Tagebuch 1941 in New York und erreichte damit Platz 1 der Bestseller-Liste der New York Times; vgl. BAP, 09.01 AA 59795. Es bildete die Grundlage für eine in deutscher Sprache gehaltene Rundfunksendung des BBC; vgl. Klee, „Euthanasie“, S. 258; BAP, 09.01 AA 69680, Bl. 144, Referent Dr. Büttner an Parteikanzlei z.Hd. Ministerialrat Krüger, 24.7. 41.

12 Shirers Informant muß über sehr engen Kontakt sowohl zum Reichsjustizminister, als auch zu Bodelschwingh und insgesamt zur Bekennenden Kirche verfügt haben. Der Informant wußte vom Protest Braunes, Bodelschwinghs und Sauerbruchs in Gürtners Privathaus, beschrieb die ambivalente Haltung des Justizministers, wußte, daß Hitler Gürtner gegenüber Reichsleiter Bouhler als den Verantwortlichen für die Aktion benannt und Bodelschwingh mit Verhaftung gedroht hatte. Er übergab ferner Todesanzeigen von „Euthanasie“-Opfern in den Leipziger Neuesten Nachrichten, die auch auf der 9. Synode der Bekennenden Kirche der Altpreußischen Union herumgereicht worden waren, vgl. Wilm, Nach der Lektüre, S. 17, und Abschriften von „Kondolenz“-Briefen mit fingierten Todesursachen an Angehörige aus Grafeneck, die auch in der Braune-Denkschrift erwähnt sind. - Shirer weiß nicht mehr, wer sein Informant war, erinnert sich bloß, daß es nicht Dohnanyi war, nwho I greatly admired [...] though he may have arranged for someone else to give it to me"; Briefliche Auskunft Shirer, 8.8. 1990. Prof. Dr. Clifford Green sei sehr herzlich dafür gedankt, daß er mir den Kontakt zu Shirer hergestellt hat.

${ }_{13}$ GS II, S. 432, Eingabe an die Wehrmacht, Oktober/November 1941.

14 Vgl. Bethge, Bonhoeffer, S. 796. Ein Protokoll ist nicht erhalten. Die Synode beschloß einen Predigtentwurf gegen den die "Euthanasie"-Aktion rechtfertigenden Film „Ich klage an" zu verfassen; vgl. Niesel, Verkündigung, S. 93,117ff. Doch sind die von Hilfsprediger Ulrich Dähne herausgegebenen „Ratschläge zur Bestattung Geisteskranker" samt „Predigtentwurf“ beschlagnahmt und Dähne verhaftet worden; vgl. Schmuhl, Rassenhygiene, S.345. Zu weiteren Verhaftungen vergleiche eine nicht unterzeichnete und nicht datierte Fürbittenliste, EZA, 50827, Bl. 157: „Hilmer geb. 1907, Verhaftet seit 6.3. 1942, nach Dachau 18.3. 42. Euthanasie ... Wilm geb. 1901. Verhaftet 23. 1. 1942, nach Dachau Ende Mai 1942. ... Über die Euthanasie gepredigt. Er hat sich mit seiner Frau und Kindern vorbereitet und gewußt, was auf die Predigt folgen kann. Lebendige Gemeinde, die jetzt tapfer durchhält".

15 Vgl. Bethge, Bonhoeffer, S.796; Hammelsbeck, Bonhoeffer, S. 144. 
achten und Protokolle. Erhalten ist lediglich ein verhältnismäßig unverfängliches Referat Bonhoeffers „Die Lehre vom primus usus legis nach den Bekenntnisschriften und ihre Kritik" vom 15. März 1943 ${ }^{16}$, in dem Bonhoeffer aufforderte, an der öffentlichen Verkündigung des Dekaloges festzuhalten: „Auch der Gemeinde in den Katakomben wird niemals die Universalität des Auftrages abgenommen [...] Niemals kann sich die Gemeinde mit der Pflege ihres eigenen inneren Lebens begnügen "17. Im Anschluß an dieses Referat erarbeitete der Ausschuß eine Synodenvorlage zu einer „Handreichung für die Ältesten und Pfarrer zum fünften Gebot" sowie ein am Dekalog orientiertes, in den Gemeinden zu verlesendes Bußtagswort für die 12. Synode der Bekennenden Kirche der Altpreußischen Union am 20.10. 1943, in welchem gegen „Euthanasie“ und Judenvernichtung Stellung genommen wurde. In der Schlußfassung heißt es:

„Das fünfte Gebot gilt immer. [...] Begriffe wie ,Ausmerzen', ,Liquidieren“ und ,unwertes Leben' kennt die göttliche Ordnung nicht. Vernichtung von Menschen, lediglich weil sie Angehörige eines Verbrechers, alt oder geisteskrank sind oder einer anderen Rasse angehören, ist keine Führung des Schwertes, das der Obrigkeit von Gott gegeben ist. [...] Unsere[r] Hilfe bedürfen auch unheilbar Kranke, Schwachsinnige und Gemütskranke. Wir haben auch ihren Familien zu helfen, die Last zu tragen. Wir dürfen auch die nicht vergessen, denen eine Hilfe aus öffentlichen Mitteln nicht oder so gut wie nicht zuteil wird. Das öffentliche Urteil hierüber kümmert den Christen nicht. Sein Nächster ist allemal der, der hilflos ist und seiner bedarf, und zwar ohne Unterschied der Rassen, Völker und Religionen. Denn das Leben ist Gott heilig. Es ist ihm heilig, auch das Leben des Volkes Israel"18. - „Wehe uns und unserem Volk, wenn das von Gott gegebene Leben für gering geachtet und der Mensch, nach dem Ebenbild Gottes erschaffen, nur nach seinem Nutzen bewertet wird; wenn es für berechtigt gilt, Menschen zu töten, weil sie für lebensunwert gelten oder einer anderen Rasse angehören, wenn Haß und Unbarmherzigkeit sich breit machen. Denn Gott spricht: ,Du sollst nicht töten "“" 19 .

Infolge seiner Verhaftung war Dietrich Bonhoeffer an der Verabschiedung der Schlußfassung auf der Synode nicht mehr beteiligt. Er hat aber noch im Gefängnis von dem Synodenbeschluß erfahren ${ }^{20}$ und ihn begrüßt.

Die spärlichen Quellen, die aus der Arbeit der Bekennenden Kirche gerettet werden konnten, zeigen auf, das Dietrich Bonhoeffer nicht nur in der eugeni-

\footnotetext{
${ }^{16}$ Der zunächst von Eberhard Bethge als Anhang zur Ethik herausgegebene Text wurde von dem Ausschußmitglied Günther Harder als Bonhoeffers Referat identifiziert; vgl. Tutzinger Texte I 1969, S. $177 \mathrm{f}$.

${ }_{17}$ Bonhoeffer, Ethik", S.335f.

${ }^{18}$ Handreichung, in: Beckmann, Kirchliches Jahrbuch 1933-1945, S.383-387.

19 Bußtagswort, in: Ebenda, S.387f.

20 Vgl. Bonhoeffer, Widerstand, S.156, Bonhoeffer an Bethge, 22.11. 1943.
} 
schen Diskussion engagiert, sondern auch in die Protestversuche gegen die „Euthanasie" involviert war. Viel stärker noch wird dies durch seine theologischen Schriften deutlich, die in der gleichen Zeit entstanden sind und in denen er sich ausführlich mit dem Thema der Eugenik und ihrer konkreten Realisation in Sterilisationspraxis und „Euthanasie“-Maßnahmen auseinandersetzt.

\section{Krankheit und Stellvertretung}

\section{Die Nähe des Gekreuzigten zum kranken Menschen}

Seine persönlichen Eindrücke im Zusammenhang mit behinderten Menschen in den Betheler Anstalten beschreibt Dietrich Bonhoeffer wie folgt:

„Die Zeit in Bethel ist für mich sehr eindrucksvoll gewesen. Es ist hier einfach noch ein Stück Kirche, die weiß, worum es einer Kirche gehen kann und worum nicht. Ich komme eben aus dem Gottesdienst: Es ist ein eigentümliches Bild, die Scharen von Epileptikern und Kranken die ganze Kirche füllen zu sehen, dazwischen die Diakonissen und Diakone, die helfen müssen, wenn irgendeiner fällt: dann wieder alte Vagabunden von der Landstraße, die Theologiestudenten, die Kinder aus der Aufbauschule für Gesunde, Ärzte und Pfarrer mit ihren Familien; aber eben doch das ganze Bild beherrscht von den Kranken, die mit einer starken Teilnahme zuhören. Es muß ja in diesen Menschen auch ein ganz eigentümliches Lebensgefühl sein, die so gar nicht Herr über sich sein können, die jeden Augenblick darauf gefaßt sein müssen, daß es sie packt. Das ging mir heute in der Kirche in solchen Augenblicken erst eigentlich auf. Und diese Situation der wirklichen Wehrlosigkeit öffnet diesen Menschen vielleicht deutlicher einen Einblick in gewisse Wirklichkeiten der menschlichen Existenz, die eben doch im Grund wehrlos[er] ist, als das uns Gesunden je gegeben sein kann. Und gerade dieser jähe Wechsel von Gesundsein und Fallen ist für solche Einsicht wohl noch verheißungsvoller als ein stetiges Kranksein. Ich mußte heute in der Kirche immer wieder an das Hundertguldenblatt von Rembrandt und die dazu gehörigen Berichte aus den Evangelien denken. Etwas Sentimentales hat das alles gar nicht, sondern etwas ungeheuer Reales, Ursprüngliches. Es fällt hier eben etwas von den Schranken, mit denen wir uns gewöhnlich von dieser Welt absperren. Sie gehört hier einfach ins eigene Leben mit hinein, wie es in Wirklichkeit ist. Von Buddha heißt es, daß er durch die Begegnung mit einem Schwerkranken bekehrt worden sei. Es ist ja ein glatter Wahnsinn, wenn man heute meint, das Kranke einfach durch Gesetze beseitigen zu können oder zu sollen. Das ist ja fast schon ein Turmbau zu Babel, der sich rächen muß. Es ist eben doch der Begriff von Krank und Gesund sehr zweideutig und daß das, was hier an ,Krankem' ist, an wesentlichen Punkten des Lebens und der Einsicht gesünder ist als das Gesunde und daß sie beide einander einfach bedürfen, das ist wohl doch eine wesentliche Gestalt und Ord- 
nung dieses Lebens, die nicht einfach frech und einsichtslos verändert werden kann" ${ }^{\text {"21. }}$.

Dieser Abschnitt eines Briefes an die Großmutter vom August 1933 sei ausführlich zitiert, weil sich hier Bonhoeffers Überlegungen zum Thema „Krankheit" zum ersten Mal verdichten und die entscheidenden theologischen Ansatzpunkte hervortreten. Bonhoeffer hat in Bethel behinderte Menschen, Epileptiker vor allem, aus der Nähe erlebt und findet sich durch die gottesdienstliche Gemeinschaft mit ihnen dahin getrieben, diese communio als ein Zeichen dafür zu verstehen, „worum es in einer Kirche gehen kann, und worum nicht“. Entscheidend ist Bonhoeffer, daß die Kranken, die sich vor einem nächsten Anfall nicht schützen können, letztlich eine intensivere Wahrnehmung der menschlichen Existenz besitzen als die Gesunden, welche die eigene Verletzlichkeit verdrängen.

In manchen dieser Überlegungen spricht er indirekt die Position des Vaters aus: daß der Übergang vom psychisch Kranken zur Norm fließend sei ${ }^{22}$, Krankheit durch $Z$ wangssterilisation nicht abgeschafft werde ${ }^{23}$ und ein prinzipieller Unterschied zwischen Geisteskrankheiten und anderen Krankheiten nicht gemacht werden könne $e^{24}$ und daß Gesunde und Kranke ihr Leben so weit wie möglich teilen sollten ${ }^{25}$. Das Erbgesundheitsgesetz wird wie beim Vater strikt abgelehnt, emotionaler noch als „Wahnsinn“ bezeichnet.

Sein Eigenes aber findet Dietrich Bonhoeffer in seiner theologischen Interpretation der Krankheit. Krankheit ist qualifiziert durch die Nähe Christi. Ein Schwerpunkt im genannten Text liegt im Verweis auf das "Hundertguldenblatt“ von Rembrandt. Dieses Bild zeigt Christus als leuchtende Gestalt inmitten der Kranken - Christus ist es, der die Schranken zwischen gesund und krank durchbricht. Was in dem Brief bildhaft über die Assoziation des „Hundertguldenblattes" beschrieben wird, hat Bonhoeffer an anderer Stelle verbaliter benannt: "Jesus wendet sich zu den Kindern und zu den moralisch und gesellschaftlich Geringen, Minderwertigen“26; und es ist „kein Zufall, daß Christus in auffallender Nähe zu den Kranken gelebt hat, daß Blinde, Gelähmte, Taubstumme, Aussätzige, Geisteskranke sich unwiderstehlich zu ihm hingezogen fühlten und seine Ge-

${ }^{21}$ GS II, S. 77f., Dietrich an Julie Bonhoeffer, 20.8. 1933. Bonhoeffer nahm diese Überlegungen 1934 in einer Abendpredigt zu 2. Kor. 12,9 wieder auf, vgl. DBW 13, S.409, und ließ für Bethel eine Kollekte sammeln, vgl. DBW 13, S. 174 f., Bonhoeffer an Fricke, 31.7. 1934.

22 Siehe S. 82.

${ }^{23}$ Siehe S. 83.

24 Siehe S. 65.

${ }^{25}$ So würdigte Karl Bonhoeffer die gescheiterte Psychiatrie-Reform seines Vorgängers Griesinger, der 1865 gefordert hatte, die "Irren" aus der "Abgeschlossenheit der Anstalten herauszuführen" und in kleineren Einheiten, sog. „klinischen Asylen“, mit Pavillons zu je 25 Personen, unterzubringen, die „im Stil von Privathäusern" erbaut werden sollten; vgl. ders., Geschichte, S. $53 \mathrm{ff}$. Bonhoeffers Aufsatz wurde erst in den siebziger Jahren im Zusammenhang mit sozialpsychiatrischen Überlegungen (Wohngemeinschaften etc.) wieder entdeckt; vgl. Güse/Schmacke, Psychiatrie I, S. 66-81; Dörner, Bürger, S.303.

26 DBW 10, S. 312 (Gemeindevortrag Barcelona 1928). 
meinschaft suchten “27. Entsprechend müssen die, die Christus nachfolgen, als „die für diese Erde ganz und gar Lebensunwerten, Überflüssigen“ erscheinen ${ }^{28}$.

In seiner Schrift Gemeinsames Leben wird dieser Gedanke theologisch und politisch zugespitzt: „Der Ausschluß des Schwachen und Unansehnlichen, des scheinbar Unbrauchbaren aus einer christlichen Lebensgemeinschaft kann geradezu den Ausschluß Christi, der in dem armen Bruder an die Tür klopft, bedeu-

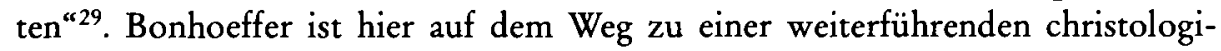
schen These: Der Heiland der Kranken, der Herr über die Krankheit, ist nicht der unberührbare Wunderheiler, sondern einer, der sich mit Kindern, Schwachen, Geisteskranken solidarisiert ${ }^{30}$, indem er ihr Leben teilt. Der Heiland ist keine glanzvolle, weltentrückte und erhöhte Gestalt, sondern ist der „Herr als Knecht “31, hat selbst teil an der leidvollen Situation der Kranken.

Bonhoeffer hat diesen christologischen Grundgedanken auch einmal ansatzweise systematisch ausgeführt, und zwar in der Finkenwalder Seelsorge-Vorlesung (1936/40): „Wenn Jesus inmitten der Kranken ist, so heißt das, daß er das Gesetz dieser Welt getragen und erfüllt hat. ,Er hat unsere Schwachheiten auf sich genommen, und unsere Sünden hat er getragen' (Matth. 8,17). Jesus heilt, indem er trägt. Sein Heilen hat nichts zu tun mit magischem, dabei aber distanziertem Gesundmachen. In seinem Heilen wird die Kreuzestat vorgezeichnet. Heilen bedeutet bei Jesus, daß er die Kranken in ihrer Schwachheit annimmt und trägt wie er sie am Kreuz tragen wird. Nur als Gekreuzigter ist er der Heiland." Auch wenn wir diesen knappen, durch die Nachschrift noch verkürzten Ausführungen kaum mehr als Stichworte entnehmen können, ist doch zumindest der christologische Ansatzpunkt deutlich: „Heiland“ kann nur derjenige sein, der selbst Mitleidender ist. Bonhoeffer interpretiert hier das Kreuzesleiden Christi im Sinne der uneingeschränkten Anteilnahme Gottes an der Schwäche und Krankheit der Menschen als dem „Gesetz dieser Welt“. Insofern findet sich jede Krankheit eingebunden in die Solidarität des Gekreuzigten. Daß der „Herr als Knecht" unter den schwächsten, bedrohtesten Menschen lebt, verändert nun auch die Welt-Wahrnehmung der Gesunden: „Unter Kranken sind wir dem

${ }^{27}$ GS III, S. 426-430 (Der beste Arzt). Auf den engen zeitlichen und sachlichen Zusammenhang mit der „Euthanasie“-Aktion machte zuerst William J.Peck aufmerksam; vgl. ders., Euthanasia-Text, S. 145.

${ }^{28}$ DBW 4 (Nachfolge), S. 110.

${ }^{29}$ DBW 5, S.33. Kodalle, Bonhoeffer, S. 54, empfindet die von Bonhoeffer mit dem Wort „kann“ ausgedrückte Einschränkung als "kompromißlerisch“. Im Rahmen von Bonhoeffers Christologie besagt sie aber nichts anderes, als daß Bonhoeffer auch dort die Gegenwart Christi nicht schlechterdings ausschließen will, wo Christus von Menschen ausgeschlossen wird.

${ }^{30}$ Bonhoeffer hat "Solidarität" von der christologischen "Stellvertretung" zunächst scharf unterschieden: „Nicht ,Solidarität', die zwischen Christus und Mensch nie möglich ist, sondern Stellvertretung ist das Lebensprinzip der neuen Menschheit ${ }^{\alpha}$; vgl. DBW 1 (Sanctorum Communio), S. 92. Für seine späteren Schriften ist bemerkenswert, „wie wichtig Bonhoeffer ... das solidarische Mitleiden ist"; Abromeit, Geheimnis, S. 285. Wir meinen daher den Begriff Solidarität unbefangen verwenden zu können, solange die Priorität Christi in diesem Geschehen klar bezeichnet ist.

${ }^{31}$ Dieser Grundgedanke Barths trifft hier sachlich zu; vgl. ders., Kirchliche Dogmatik IV/1, S. $171 \mathrm{ff}$. 
Kreuzesleiden Jesu näher und erkennen die Welt besser als unter Gesunden. [...] Unsere Gesundheit ist in jedem Augenblick gefährdet. Alle Krankheit ist in unserer Gesundheit immer schon enthalten. Das Gesetz der Welt heißt Kreuz, nicht Gesundheit. Es ist nicht gut, die Kranken durch Konzentrierung in großen Kliniken dem Blick der Gesunden immer mehr zu entziehen. In Bethel leben Kranke und Gesunde in großer Selbstverständlichkeit miteinander, im Alltag und im Gottesdienst: ein dauerndes Erinnern der Gesunden an die Kranken “32.

Ein weiterer Text aus dem Jahr 1941, „Der beste Arzt“, bespricht das Verhältnis von Krankheit und Glaube, somatischer und spiritueller Erkrankung und Gesundung. In einem klassisch-lutherischen Argumentationsweg, im Schema von Gesetz und Evangelium, deutet Bonhoeffer Krankheit als Signal für eine gestörte Gottesbeziehung, die aber damit den Rückweg zu Gott vorbereiten helfen kann ein Weg, der durch die Beichte vollzogen wird. Abgesehen von höchst modernen Überlegungen zur Psychosomatik erscheint dieser Text sehr hölzern und die oben aufgeführten Äußerungen durchaus kontrastierend, insofern Krankheit wohl nicht als Sünde selbst erscheint, aber als Hinweis darauf: „Es ist die Sünde der Welt und es ist meine eigene Sünde, an die ich erinnert werde. Meine Krankheit braucht nicht einfach eine Folge oder Strafe einer bestimmten Sünde zu sein, deren ich mich anzuklagen hätte - auch dies mag der Fall sein -, es ist aber nicht notwendig so. Doch will mich jede Krankheit in die Tiefe der Weltsünde und meiner persönlichen Gottlosigkeit hineinblicken lassen " ${ }^{33}$. Unheilbare Krankheit, und damit das Thema der geistigen und körperlichen Behinderung, wird nicht eigens in Blick genommen. Auf diese erstaunliche Tatsache kann man sich nur schwer einen Reim machen; umso weniger, als Bonhoeffer mit jenem Aufsatz die Arbeit am Thema „Euthanasie“ unterbrach, mit dem er vorrangig beschäftigt war ${ }^{34}$. Daß mit dem Beitrag für die Bädermission ein sehr spezieller Adressatenkreis angesprochen ist, gibt m. E. keine hinreichende Antwort.

Ein Gedanke dieses Textes ist jedoch systematisch weiterführend: daß eine Krankheit den Verlust des eigenen, menschlichen Herrschaftswillens und die Anerkennung einer fremden Macht, der liebenden Herrschaft Gottes, impliziert. Dieses Motiv schließt sich mit dem obengenannten Gedanken zusammen, daß Herrschaft Gottes nicht nach menschlichen Maßstäben gemessen wird, vielmehr in der Ohnmacht ihre eigentliche Gestalt und Kraft gewinnt.

"Krankheit" dürfte für den Fünfunddreißigjährigen in dieser Zeit noch nicht eigentlich zu einem existentiellen Thema geworden sein - eigene Krankheiten duldete er gar nicht gern und bekämpfte sie energisch und großzügig mit Medikamenten ${ }^{35}$. Jedoch vermag er unbeschadet dessen zu diesem Thema einen Rahmen der theologischen Reflexion zu entwickeln, den er später weiterführt und

\footnotetext{
32 Vgl. GS V, S. 392.

33 GS III, S. 428.

34 Vgl. GS II, S.394f., Bonhoeffer an Bethge, o.D.

35 Vgl. Bethge, Bonhoeffer, S. 19.
} 
näher ausfüllt. Entscheidend ist der christologische Ansatzpunkt: Der „Heiland“ Jesus Christus ist nicht ein der Krankheit enthobener Wundertäter, sondern Gott identifiziert sich in der Menschwerdung mit der Krankheit als mit der verlorenen Welt. Für die gesunden Menschen folgt daraus ein neues Erkenntnisprinzip: Krankheit ist dieser Welt unmittelbar zugehörig, sie kann nicht ausgegliedert oder gar abgeschafft werden. Kranke Menschen sind Hinweise auf die faktische Labilität der menschlichen Existenz. Mehr noch: Sie sind Zeichen der Parteinahme Gottes für das Niedriggeachtete.

Echte Wirklichkeitserkenntnis gelingt dem Menschen daher nur, wenn die Krankheit, auch die unheilbare, in das Weltbild mit einbezogen wird. Anhand der Krankheit geht es darum, den menschlichen Herrschaftswillen und Anspruch des gesunden Menschen als ambivalent zu begreifen. „Das Gesetz der

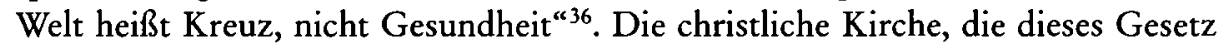
erkennt, muß darum alles tun, kranke Menschen zu ehren und zu schützen.

Der Grundgedanke, daß in der Nachfolge Christi nur derjenige wirklich Mensch sein kann, der die Krankheit und die Kranken als Teil der Lebenswirklichkeit zu begreifen versteht, wird in Bonhoeffers Verantwortungsethik fortgeführt. Bezüglich der medizinischen Ethik gilt diese These in der Weise, daß nur derjenige den Beruf des Arztes verantwortlich ausüben kann, der die Kranken als Menschen versteht, deren Würde er achtet.

\section{Berufliches (ärztliches) Handeln als verantwortungsethisches Handeln}

Dabei soll nun zunächst Bonhoeffers Begriff vom „Beruf“ und damit sein verantwortungsethischer Ansatz näher beleuchtet werden. Wir beziehen uns im folgenden auf zwei grundlegende Abschnitte der Ethik: „Die Struktur verantwortlichen Lebens" und „Der Ort der Verantwortung" als Bestandteil der zweiten Fassung von „Die Geschichte und das Gute" (S. 245-299) ${ }^{37}$.

Bonhoeffers Konzept der Verantwortungsethik läßt sich durch eine Gegenüberstellung mit der Position Max Webers, auf den er sich ausdrücklich bezieht, besonders klar herausarbeiten (S. 254) ${ }^{38}$; denn Max Weber ist der einzige, der bereits vor Bonhoeffer eine explizite Verantwortungsethik formuliert hatte. Im Vergleich der beiden Positionen ${ }^{39}$ werden die Unterschiede sichtbar:

36 GS V, S. 392.

37 Seitenangaben für die Ethik finden sich (um der Übersicht willen), nachfolgend im Text (nach DBW 6).

38 Als zweites Beispiel führt Bonhoeffer Bismarck an. Es findet sich bei Baumgarten, Politik, S.110f., ein Buch, auf das Bonhoeffer in diesem Kapitel verschiedentlich anspielt; vgl. die Anmerkungen der Herausgeber zu DBW 6 (Ethik). Baumgarten, ein Vetter Max Webers, betont ähnlich wie jener den an-ethischen Charakter der Politik und sieht die Unterordnung der „Privatmoral der Bergpredigt“ unter das "nationale Ethos“ bei Bismarck ideal vorgebildet; vgl. ebenda, S.112. Ein solcher, politisch an Bismarck orientierter Liberalismus hat erst jüngst wieder Bewunderer gefunden, vgl. Brakelmann, Krieg.

39 Vgl. dazu durchgängig auch Huber, Sozialethik S. 138-143. 
Während Weber bei dem für andere verantwortlichen einzelnen ansetzt ${ }^{40}$, macht Bonhoeffer den Begriff „verantwortliches Leben“ (analog zu dem unten zu behandelnden "natürlichen Leben“) zum zentralen Begriff seiner Verantwortungsethik, denn der ,isolierte einzelne“ als Subjekt allen ethischen Handelns ist eine „Fiktion“ (S.220). Bonhoeffer definiert Verantwortung als „die zusammengefaßte Ganzheit und Einheit der Antwort auf die uns in Jesus Christus gegebene Wirklichkeit" (S.254). Indem er den christlichen Glauben verbunden sieht mit der in Christus gegebenen Wirklichkeit, qualifiziert er ihn nicht als eine bloße Gesinnung. Der von Weber aufgebaute - freilich auch nicht absolut gemeinte - Gegensatz zwischen Gesinnungs- und Verantwortungsethik wird so vermieden. Terminologisch trägt Bonhoeffer dem Rechnung, indem er der Gesinnungsethik die „Erfolgsethik“ entgegensetzt (S.37), die ohne Rücksicht auf die Wahl der Mittel allein auf den Zweck abzielt. Gesinnungs- und Erfolgsethik seien gleichermaßen an einem von der Wirklichkeit losgelösten Begriff des Guten orientiert - die eine, indem sie das Gute durch die "Gesinnung" (und nicht durch Mittel und Erfolg), die andere, indem sie das Gute durch den Erfolg (und nicht durch "Gesinnung" und Mittel) bestimmt sein läßt. Die Verantwortungsethik dagegen orientiert sich am Ganzen der in Christus gegebenen Wirklichkeit. Mit diesem christologisch bestimmten Wirklichkeitsverständnis widerspricht Bonhoeffer einer von Weber geforderten reinlichen Scheidung zwischen "Realität" und „Werten“"1, so wie er es überhaupt ablehnt, das Gesollte gegen das Seiende und das Seiende gegen das Gesollte auszuspielen (S.39).

Von dem Kapitel „Der Ort der Verantwortung“ (S.289-299) ist lediglich der Abschnitt über den Beruf erhalten. Im Anschluß an Webers Aufsatz Die protestantische Ethik und der Geist des Kapitalismus ${ }^{42}$ vertritt Bonhoeffer die Ansicht, daß Luthers Übersetzung mit dem für die deutsche Sprache neugeschaffenen Wort „Beruf“ für „klesis“ in 1. Korinther 7,20 (sonst auch mit „Ruf“ oder „Berufung“ übersetzt) über den neutestamentlichen Sprachgebrauch hinaus-

40 Vgl. Weber, Politik, S. 548.

${ }^{41}$ Die soziologische Problematik dieser Trennung wird dort deutlich, wo erklärt werden soll, wie der Soziologe ohne Bezugnahme auf "Werte“ überhaupt entscheiden kann, welchem von der Unzahl der zu erforschenden Themen er sich überhaupt zuwenden solle. Weber beantwortet sie durch Hinweis auf die "Richtung unseres Erkenntnisinteresses, wie sie sich aus der spezifischen Kulturbedeutung ergibt, die wir dem betreffenden Vorgang im einzelnen Fall beilegen “. Er belegt das damit, daß der Soziologe gleichermaßen der Religion, der Prostitution und dem Geld eine "Kulturbedeutung" zuschreiben könne, ohne Aussagen darüber machen zu müssen, welchen positiven oder negativen "Wert" diese Erscheinungen für ihn hätten; vgl. Weber, Objektivität, S.161, 181. Die Debatte seit dem sog. Positivismus-Streit der sechziger Jahre hat ergeben, daß diese Unterscheidung problematisch ist, weil es nicht nur binär kodierbare Wertbegriffe im Sinne Luhmanns gibt (wie etwa "gut ${ }^{*}$ - "böse“, "begrüßenswert" - „ablehnenswert"), sondern auch Wertbegriffe zweiter Ordnung, zu denen „Erkenntnisinteresse ${ }^{\alpha}$ und „Kulturbedeutung“ gehören. Beide können keineswegs als "wertfreie" Begriffe angesehen werden. - Bonhoeffer wäre im PositivismusStreit „zwischen die Fronten“ geraten. So zu Recht Feil, Theologie, S. 87.

${ }^{42} \mathrm{Vgl}$. Weber, Protestantische Ethik, S. 68. 
gehe ${ }^{43}$, sieht dies zugleich aber als „sachlich gerechtfertigt“ an. Allerdings dürfe man nicht - wie das „Pseudoluthertum" - im Beruf eine "Rechtfertigung und Heiligung der weltlichen Ordnungen an sich ${ }^{\text {“44 }}$ erblicken (S. 290). In Webers Definition des Berufes als "abgegrenztes Gebiet von Leistungen“45 würden die Grenzen beruflicher Verantwortung zu stark betont. Bonhoeffer sieht den Beruf gekennzeichnet durch die „Inanspruchnahme durch Christus an dem Ort, an dem ich von diesem Ruf getroffen wurde" (S.293). Diese Inanspruchnahme gelte gleichzeitig "nach oben“, also zu Christus hin, und "nach außen“, also in Richtung auf Mensch und Gesellschaft.

Bonhoeffer demonstriert seine Berufsauffassung, indem er an einen in den USA im Jahre 1931 geführten Prozeß erinnert, in dem „9 junge Neger" wegen Vergewaltigung eines weißen Mädchens von zweifelhaftem Ruf zum Tode verurteilt worden waren, obwohl sie ihrer Schuld nicht überführt werden konnten (S.296). Während „anerkannteste europäische Persönlichkeiten“ - ein Kreis um Albert Einstein ${ }^{46}$-, den Rahmen ihres Berufes weit spannend, gegen das Urteil öffentlich protestiert und damit die Aufhebung des Urteils erreicht hätten, habe ein anerkannter deutscher Kirchenmann (gemeint war vermutlich Otto Dibelius) sich aufgrund eines falsch verstandenen „lutherischen“ Berufsverständnisses einem Protest verweigert (S. 296 Anm. 159).

Das Beispiel markiert deutlich die politischen Konnotationen von Bonhoeffers Ausführungen. Wenn der „Beruf“ eines Menschen nicht auf Pflichterfüllung in einem abgegrenzten Bereich zu beschränken ist - so kritisch gegen Weber, schärfer noch gegen die neulutherische Zweireichelehre -, werden die Grenzen der persönlichen Verantwortung ausdrücklich weit gezogen. „Nicht in der treuen Leistung seiner irdischen Berufspflichten... erfüllt der Mensch die ihm auferlegte Verantwortung, sondern im Vernehmen des Rufes Jesus Christi, der ihn zwar auch in die irdischen Pflichten hineinführt, aber niemals in ihnen aufgeht" (S. 291). „Wir geben. .. dem Begriff der Verantwortung eine Fülle, die ihm im alltäglichen Sprachgebrauch nicht zukommt. .." (S.254), und die sich in der Tradition am ehesten an Luther orientiert ${ }^{47}$. Weitgehender noch ist die These, daß der Beruf - wenn er als Ruf Christi gehört wird - sogar hinführen kann in die Verneinung der sonst gebotenen alltäglichen Pflichten zugunsten des Protestes und

${ }^{43}$ So auch - unter Berufung auf Weber - K. L. Schmidt, klesis. Den lutherischen Sprachgebrauch schon bei Paulus vorgezeichnet sieht Holl, Geschichte, S.190.

${ }^{44}$ Bonhoeffer nennt keine Namen, vgl. aber Althaus, Grundriß, S. 67f.: „Der Christ erkennt in den Pflichten seines Berufes den Willen Gottes an ihn. [...] auch wo der Einzelne den Sinn seines Berufes als Dienst am Ganzen nicht erkennen kann oder ihn als nichtsnutzig oder objektiv sündig erkennt und doch nicht fliehen kann [. . . ] auch da gilt es, das Berufswerk im Gehorsam gegen Gott, der es mir - auch durch das Chaos und die Sünde der Gesellschaft hindurch - gab, es zu tun." Ähnlich Emil Brunner, Gebot, S.185, „Geheiligtheit des an sich Unreinen durch Gottes vergebende Berufung: das ist der Beruf".

${ }^{45}$ Vgl. Weber, Protestantische Ethik, S. 68 Anm. 1; ders., Wirtschaft, S. 80.

${ }^{46}$ Bonhoeffer nennt keine Namen. Zu Einsteins Bemühungen vgl. aber Grüning, Haus, S. 353.

47 Vgl. dazu die Belege bei Holl, Beruf, S. 213-219. 
Widerstands. In jedem Fall steht die unbedingte Verpflichtung der individuellen Verantwortung an das Gemeinwohl außer Frage: „Im Hinblick auf Bonhoeffer lassen sich Verantwortungsethik und Sozialethik in der Tat nur als Synonyme verstehen. [...] Nicht die individuelle Lebensführung für sich, sondern der Beitrag der individuellen Lebensführung zur Gestaltung der geschichtlichen Gegenwart ist das Thema der Ethik ${ }^{\star 48}$.

In seiner Erklärung der „Struktur verantwortlichen Lebens“ mit Hilfe der vier Begriffe „Stellvertretung", „Wirklichkeitsgemäßheit“, „Schuldübernahme“ und „Freiheit ${ }^{\star 49}$ entfaltet Bonhoeffer die Grundlagen für sein anspruchsvolles und dichtgefügtes ethisches Konzept. Das Freiheitsmoment, das Gestalt gewinnt als Wagnis, als Ausliefern der Tat und als Offenbleiben der Sinnfrage, ist für Bonhoeffer nicht denkbar ohne die drei Bindungsmomente Stellvertretung, Wirklichkeitsgemäßheit und Schuldübernahme. Wir referieren diese Grundlegung im folgenden mit Blick auf die Richtungsweisung, die Bonhoeffers Ansatz für das ärztliche Handeln bzw. die Arzt-Patient-Beziehung ergeben könnte. Teilweise hat Bonhoeffer auch selbst Beispiele für diesen Bereich ausformuliert.

Die Stellvertretung ${ }^{50}$ Christi begründet die zwischenmenschliche Verantwortlichkeit. Abermals gewinnt Bonhoeffer seine Kriterien in der streng christologischen Grundlegung: der Menschgewordene ist der Urgrund jeder menschlichen Stellvertretung, sein Eintreten für die Menschen begründet die Verantwortlichkeit einzelner Menschen für andere. Bonhoeffer nennt für die zwischenmenschliche Stellvertretung das Beispiel des Vaters, Staatsmannes und Lehrers, dem läßt sich das des Arztes/der Ärztin zwanglos hinzufügen. Die Vollmacht des (ärzt-

\footnotetext{
${ }^{48}$ Huber, Sozialethik, S. 143.

49 Bonhoeffer hat die Gliederung noch während des Schreibens verändert. Er kündigt in der Einleitung (S. 256) andere Leitbegriffe an, als er später ausführt und am Ende zusammenfaßt (S.289). Ich folge der von Bonhoeffer tatsächlich durchgeführten Gliederung.

${ }^{50}$ Bonhoeffer hat den traditionellen Begriff der "Stellvertretung" in seiner Theologie an zentrale Stelle gerückt: bereits in Sanctorum Communio als Leitbegriff eingeführt, bleibt er in den nachfolgenden Schriften relevant als derjenige Terminus, „der es ihm ermöglicht, Christologie, Ekklesiologie und Ethik theologisch begründet zusammenzuhalten"; vgl. Soosten, Nachwort zu DBW 1, S.321. Bonhoeffers Übernahme des traditionell christologischen Gedankens in die Ekklesiologie und Ethik hat verschiedentlich kritische Kommentare evoziert; Übersichten über diese Diskussion bei Dinger, Bonhoeffer, S. 8; Abromeit, Geheimnis, S.268 Anm. 1, S.284, wobei eine gründliche Untersuchung aber deutlich macht, daß eine Unterscheidung zwischen "theologischer" und "anthropologischer" Verwendung des Begriffs jedenfalls nicht in Bonhoeffers Sinn ist, so mit Abromeit, Geheimnis, S. $268 \mathrm{ff}$., gegen Jüngel, Geheimnis, S. 20. Wichtig scheint mir hingegen, daß die christologische Basis des Stellvertretungsgedankens, auf die Bonhoeffer in keinem Fall hätte verzichten können, anders Sölle, Stellvertretung, S.123, in der Ethik anders formuliert wird als in den früheren Schriften. Das stellvertretende Strafleiden Christi kommt nicht zur Sprache, hingegen geschieht "Stellvertretung" bereits in der Inkarnation: "Christus wurde Mensch und trug damit stellvertretende Verantwortung für Menschen"; vgl. DBW 6 (Ethik), S. 259. Es entspricht Bonhoeffers Hinwendung zum "Vorletzten", wenn die traditionelle soteriologische Verwendung des Begriffs (so auch noch ausgeprägt in Sanctorum Communio) in der Ethik ergänzt wird durch eine „Stellvertretung des Lebens“: „Weil Jesus - das Leben, unser Leben, - als der Menschgewordene Sohn Gottes stellvertretend für uns gelebt hat, darum ist alles menschliche Leben durch ihn wesentlich stellvertretendes Leben." DBW 6 (Ethik), S.257.
} 
lich) Verantwortlichen reicht immer nur so weit, wie er "das Ich mehrerer Menschen in sich vereinigt" (S.257), d.h. das Anliegen der Patienten/innen voll und ganz zu dem seinigen macht. Eine Absolutsetzung des eigenen Ich würde zur Tyrannei gegenüber dem Nächsten, eine des anderen Ich zu Willkür und Orientierungslosigkeit führen (S.260). Um Christi willen steht die Orientierung am Menschen im Mittelpunkt, welche durch keine "Sachzwänge“ gebrochen werden darf (S. 259).

Wie die menschliche Stellvertretung für andere fern von aller Selbstherrlichkeit "das Ich mehrerer Personen“ in sich tragen muß, so findet sie auch ihre Grenze an der Verantwortlichkeit des anderen Menschen. „Die Verantwortung des Vater oder des Staatsmannes ist begrenzt durch die Verantwortlichkeit des Kindes oder des Staatsbürgers, ja es besteht die Verantwortung des Vaters oder des Staatsmannes ebendarin, die Verantwortlichkeit der ihm Anbefohlenen ins Bewußtsein zu heben, zu stärken“. (S. 269) Somit beruht Verantwortungsethik auf Gegenseitigkeit und akzentuiert doch, so resümiert Huber, ein „Moment der Einseitigkeit, des Zuvorkommens, das nicht vollständig an die Bedingungen der Gegenseitigkeit gebunden ist“51.

Die Anerkenntnis der Begrenztheit, so Bonhoeffer, ist ein Aspekt der Wirklichkeitsgemäßheit des verantwortlichen Handelns. Der verantwortlich Handelnde sucht nicht das "absolut Gute" zu verwirklichen, welches gerade das Schlechteste sein kann, sondern wird in Selbstbescheidung das relativ Bessere dem relativ Schlechteren vorziehen (S.260). Die letzte Rechtfertigung menschlichen Tuns liegt ohnehin nicht in den eigenen Händen, noch in irgendeinem noch so positiven Prinzip. Wirklichkeitsgemäßheit gründet im Wirklichen selbst, dessen Name Jesus Christus heißt. Im Ruf Christi aber ist der Mensch an diese Welt, mit all ihren Ambivalenzen, und an seinen konkreten Verantwortungsbereich gewiesen. Als ethisch-verantwortlich gilt eine Haltung, die sich von allgemeinen moralischen Prinzipien zu trennen vermag, um dem Gebot Gottes in der konkreten Situation folgen zu können, und die damit auf Selbstrechtfertigung verzichtet, um das Urteil einem anderen zu überlassen. Wirklichkeitsgemäß handelt, wer ohne frommen Eifer, aber mit vollem Einsatz seine Aufgabe ausfüllt: „Kein Mensch hat den Auftrag, die Welt zu überspringen und aus ihr das Reich Gottes zu machen." (S.266)

Damit ist nicht eine Begrenzung der, wie oben deutlich wurde, grundsätzlich weit gefaßten Verantwortung impliziert, wohl aber ein Verweis an die konkrete Situation: „Nicht die Welt aus den Angeln zu heben, sondern am gegebenen Ort das im Blick auf die Wirklichkeit Notwendige zu tun, kann die Aufgabe sein." (S. 267) „Es wird [...] der Mensch in die konkrete und damit begrenzte geschaffene Verantwortung gestellt, die die Welt als von Gott geliebte, gerichtete und versöhnte erkennt und dementsprechend in ihr handelt. Die, Welt ist so der uns in und durch Jesus Christus gegebene Bereich der konkreten Verantwortung[... . “ (S. 266).

${ }^{51}$ Vgl. Huber, Sozialethik, S. 144. 
Diese Überlegungen Dietrich Bonhoeffers lassen sich, in und mit ihrer theologisch klaren Fundierung, zugleich als Reverenz an den Vater lesen, dessen Arbeit sich für den Sohn eben darin auszeichnen mochte, daß der Arzt und Wissenschaftler sich auf das Seine zu bescheiden vermochte, in seinem Bereich der „konkreten Verantwortung" aber alle Möglichkeiten wahrnahm und ausfüllte, die ihm zu Gebote standen - bis hin zum regimekritischen Engagement. So schreibt Bonhoeffer in seinen Ausführungen zum „Beruf“: „Bin ich zum Beispiel Mediziner, so diene ich nicht nur im konkreten Falle meinen Patienten, sondern zugleich der naturwissenschaftlichen Erkenntnis und damit der Wissenschaft und der Wahrheitserkenntnis überhaupt. Obgleich ich praktisch diesen Dienst an meiner konkreten Stelle, also zum Beispiel am Krankenbett des Patienten, versehe, so behalte ich doch die Verantwortung für das Ganze im Auge und nur so erfülle ich meinen Beruf. Dabei kann es dazu kommen, daß ich als Arzt auch meine konkrete Verantwortung einmal nicht am Krankenbett, sondern zum Beispiel im öffentlichen Eintreten gegen eine die medizinische Wissenschaft oder das menschliche Leben oder die Wissenschaft als solche bedrohende Maßnahme erkennen und erfüllen muß. Gerade weil Beruf Verantwortung ist und weil Verantwortung eine ganze Antwort des ganzen Menschen auf das Ganze der Wirklichkeit ist, gibt es kein banausisches Sichbeschränken auf die engste Berufspflicht; eine derartige Beschränkung wäre Verantwortungslosigkeit. Wann und in welchem Umfang ein solches Durchbrechen des, abgegrenzten Gebietes von Leistungen' zum Beruf und zur Verantwortung des Menschen gehört, läßt sich um des Wesens der freien Verantwortung willen in keine gesetzlichen Bestimmungen fassen. Es wird nur unmittelbar geschehen können nach ernster Abwägung der unmittelbar gegebenen Berufspflicht, der Gefahren des Eingreifens in fremde Verantwortlichkeit und schließlich des Ganzen, um das es geht; es wird dann eine freie Verantwortung gegenüber dem Ruf Christi sein, die mich hierhin und dorthin führt. Die Verantwortung im Beruf folgt allein dem Ruf Christi. Es gibt eine falsche und eine richtige Beschränkung und eine falsche und eine richtige Ausweitung der Verantwortung, es gibt ein schwärmerisches Durchbrechen aller Grenzen und ein gesetzliches Aufrichten von Grenzen. So schwierig oder unmöglich es ist, von außen her zu beurteilen, ob in einem konkreten Fall verantwortlich oder schwärmerisch bzw. gesetzlich gehandelt wird, so gibt es doch Maßstäbe zur Selbstprüfung, ohne daß allerdings auch diese zu einer vollen Gewißheit über das eigene Ich führen könnten. Solche Maßstäbe sind: weder die Begrenzung noch die Ausweitung meiner Verantwortlichkeit darf prinzipiell begründet sein, ihre einzige Begründung kann der konkrete Ruf Jesu sein; wo ich mich in meiner charakteristischen Anlage als reformerisch, besserwisserisch, fanatisch, grenzenlos kenne, dort werde ich in der Gefahr sein, meine Verantwortung willkürlich auszuweiten und meine natürlichen Triebe mit dem Ruf Jesu zu verwechseln; wo ich mich als vorsichtig, ängstlich, unsicher und gesetzlich kenne, dort werde ich mich hüten müssen, die Begrenzung meiner Verantwortung auf einen engen Bereich als Ruf Jesu auszugeben; schließlich kann niemals 
der Blick auf mich selbst, sondern allein der Blick auf den Ruf Christi mich zur echten Verantwortung befreien." (S.294f.)

Bonhoeffer bringt das Beispiel des Arztes als Beleg für eine Berufstätigkeit ein, die in ihren unterschiedlichen Aufgabenbereichen - vorrangig Dienst am Krankenbett, aber gleichfalls im Wissenschaftsbereich oder auch einmal in der öffentlichen Stellungnahme - jeweils Verantwortung wahrnimmt. Das Beispiel des Arztes macht deutlich, daß es unverantwortlich wäre, sich nur auf einen Sektor oder einen enggezogen Kodex von Pflichten zu beschränken. Wenn Bonhoeffer den Verantwortungsbegriff betont weit faßt, so wendet er sich damit auch gegen die Scheidung „zweier Reiche“, etwa als Trennung zwischen berufsspezifischem und politischem Handeln. Vielmehr bindet er beides aneinander und spitzt seine These mit Blick auf die tagespolitischen Ereignisse zu: daß der Arzt seine Pflicht auch einmal „im öffentlichen Eintreten gegen eine die medizinische Wissenschaft oder das menschliche Leben oder die Wissenschaft als solche bedrohende Maßnahme erkennen und erfüllen muß".

Wenn nun aber der Bereich der Verantwortung grundsätzlich so weit gespannt ist, besteht die Gefahr, daß über einer übereifrigen Verantwortung für das Ganze („schwärmerisch“) die konkrete Verpflichtung verlorengeht. Bonhoeffer verlangt hier zum einen präzise Selbstreflexion - wer sich eher als großspurig und fanatisch kennengelernt hat, möge seine Grenzen eher eng setzen, und umgekehrt -, zum anderen wird grundsätzlich dem moralischen Prinzipientum gewehrt, welches weder der Begrenzung noch der Ausweitung der Berufspflicht zugrunde liegen darf. "Ihre einzige Begründung kann der konkrete Ruf Jesu sein." Insofern kommt die reflexive Selbsterkenntnis an ihre Grenze, als letztlich nur die Anrede Gottes an den Menschen, und damit der Blick des Menschen über sich und seine Prinzipien hinaus, wirklich Freiheit und Verantwortlichkeit stiften kann.

\section{„Sachgemäßheit " der Wissenschaft}

Das Beispiel ärztlichen Handelns macht weiterhin deutlich, daß, wie oben schon herausgestellt wurde, für das ethische Handeln die Orientierung am Menschen im Vordergrund steht. Dennoch räumt Bonhoeffer der Wissenschaft gleichzeitig einen hohen Stellenwert ein. Wie ist das Verhältnis beider Bereiche zueinander zu denken?

In Bonhoeffers Ausführungen zur „Wirklichkeitsgemäßheit“ ethischen Handelns, von denen wir ausgegangen waren, wird der Zusammenhang zwischen der (primären) Verantwortung für die Menschen und der Verantwortung für den „Bereich der Dinge“ (S.269) unter dem Kriterium der "Sachgemäßheit“ verhandelt.

Damit ist dasjenige Verhalten zu den Dingen gemeint, welches „ihre ursprüngliche, wesenhafte und zielhafte Beziehung auf Gott und den Menschen im Auge behält" (S. 270) und zugleich das "Wesensgesetz" einer „Sache" (etwa mathemati- 
scher Axiome, der Logik, des Staates, der Familie, einer Fabrik oder einer Aktiengesellschaft) berücksichtigt (S.271). Diese Beispiele sind nicht zufällig gerade denjenigen Lebensbereichen entnommen, denen Max Weber eine „Eigengesetzlichkeit" zuschrieb ${ }^{52}$. Bonhoeffer nimmt dabei Webers Anliegen auf, Sachgesichtspunkte zu berücksichtigen, verlangt aber im Unterschied zu Weber, daß bei allen Sachgesichtspunkten die "wesenhafte und zielhafte Beziehung auf Gott und den Menschen" berücksichtigt werden müsse. Es kann daher nach Bonhoeffer keine Sachgesichtspunkte geben, die dem Menschen schaden. Bonhoeffer erläutert den Wert „sachgemäßen“ Handels am Beispiel der Wissenschaft: „Je reiner, je freier von persönlichen Nebenzwecken einer Sache gedient wird, desto mehr gewinnt sie ihre ursprüngliche Beziehung zu Gott zurück, desto mehr befreit sie den Menschen von sich selbst. Die Sache, der das letzte persönliche Opfer gebracht wird, muß gerade so den Menschen dienen. Wo zum Beispiel eine Wissenschaft aus demagogischen, pädagogischen, moralistischen Gründen in fälschlich direkter Weise dem Menschen nutzbar gemacht werden soll, dort wird nicht nur der Mensch, sondern auch die Wissenschaft verdorben. Wo aber der Mensch in der Wissenschaft allein und vorbehaltlos der Erkenntnis der Wahrheit dient, dort findet er in der selbstlosen Preisgabe aller eigenen Wünsche sich selbst, und die Sache, der er selbstlos diente, muß zuletzt ihm dienen." (S. 270)

Bonhoeffer begreift die Arbeit an einem Sachzusammenhang als befreiendes Gegenüber, das der Selbstbezogenheit des Menschen gesetzt ist. Insofern dient die Arbeit der Befreiung von eigenen fixen Prinzipien und öffnet die Bereitschaft, den konkreten Ruf Gottes zu hören. Diese theologisch tiefe Wertschätzung der Arbeit, die Luther nachdrücklich formuliert hat ${ }^{53}$, erscheint bei Bonhoeffer bereits in Sanctorum Communio ${ }^{54}$, wird hier nun aber ungleich konkreter und präziser zur Sprache gebracht. Wenn Bonhoeffer zum Kriterium wirklichkeitsgemäßen Handelns neben der Anerkennung der eigenen Begrenzung die „Sachgemäßheit" und die Anerkennung von „Wesensgesetzen“ erhebt, bringt er damit zum Ausdruck, daß Personalethos und Sachethos ineinander verschlungen sind $d^{55}$ - so wie auch in der Medizin der kranke Mensch als Person mit der Krankheit als wissenschaftlich zu untersuchendes Phänomen verbunden ist. Diese Sichtweise bringt Bonhoeffer zugleich kritisch gegen die nationalsozialistischen Eingriffe in den Wissenschaftsbetrieb zur Geltung, in denen er einen falschen Versuch sieht, die Wissenschaft dem Menschen aus „demagogischen, pädagogischen, moralistischen Gründen" in "fälschlich direkter" Weise nutzbar zu machen und dabei doch sowohl dem Menschen als auch der Wissenschaft zu schaden. Wir erinnern in diesem Zusammenhang daran, daß beispielsweise die

\footnotetext{
52 Vgl. Weber, Zwischenbetrachtung, in: Religionssoziologie I, S. 544-565.

53 Vgl. Holl, Beruf, S. 213-219.

54 Vgl. DBW 1, S. 122.

55 Vgl. seine Auseinandersetzung mit Dilschneider; Bonhoeffer, Personalethos und Sachethos, in: ders., Ethik ${ }^{11}$, S. 341-352.
} 
"Gleichschaltung“" psychiatrischer und neurologischer Vereine mit den „gemeinsamen Aufgaben“ gerechtfertigt wurde, die sie „z.B. auf dem Gebiete der Erbkrankheiten zu lösen haben " 56 , sich also durchaus als scheinbar wohlmeinende Indienstnahme für einen praktischen $Z$ weck verstand.

\section{Schuldübernabme und Freibeit}

Den bisher entwickelten Überlegungen entsprechen die beiden nachfolgenden Strukturmomente verantwortlichen Lebens: Schuldübernahme und Freiheit. Das letztgenannte Moment steht deutlich in der Traditionslinie christlicher Ethik wahre Freiheit wird als „Bindung an Gott und den Nächsten“ verstanden $(\text { S.283) })^{57}$; von jeglicher Bindung losgelöst, stellt sie sich als Akt der Willkür dar (S.288). Dagegen zeigen Bonhoeffers Ausführungen zur "Schuldübernahme" wohl am stärksten die zeitgeschichtliche Konnotation. Man muß sie zunächst im Kontext der speziellen biographischen Erfahrung lesen, daß der Theologe Bonhoeffer, wie alle Verbündeten des Widerstandskreises, sich täglich mit Lügen wappnen mußte, um überleben und um seinen Einsatz weiterführen zu können.

Bonhoeffer bindet diese Erfahrungen in seine grundlegenden Überlegungen zur Schuldübernahme in eine sehr weitreichende und auch provokante christologische Begründung ein: Christus tritt im umfassenden Sinne in die Schuld der Menschen ein. Er, der Sündlose, ist als geschichtlich und verantwortlich Handelnder schuldig geworden, etwa indem er aus Liebe das Sabbathgesetz brach, seine Eltern verließ und mit Sündern und Verworfenen aß (S. 279). In diesem schuldlos-sündigen Handeln Jesu hat jedes stellvertretende verantwortliche Handeln seinen Ursprung. Wer meint, sich mittels eines durchzuhaltenden ethischen Prinzips der Schuld entziehen zu können, handelt verantwortungslos und verweigert sich dem Gebot der Nächstenliebe. Für Jesus hingegen ging es nach Bonhoeffer eben "nicht um die Proklamation und Verwirklichung neuer ethischer Ideale, also auch nicht um sein eigenes Gutsein (Mt 19!), sondern allein um die Liebe zum wirklichen Menschen" ${ }^{\text {"58. }}$ - Wir werden auf diesen Gedankenkomplex und seine Konsequenzen für die medizinische Ethik im folgenden noch näher eingehen ${ }^{59}$.

56 Siehe S.71.

57 Vgl. Luther, Werke Bd.7, S. 20-38 (Freiheit eines Christenmenschen).

58 Mit diesem Ansatz widerspricht Bonhoeffer jeglicher „Gesinnungsethik“. Er zitiert und kritisiert das klassische Beispiel Kants, dessen Forderung, man müsse selbst einem in ein Haus eingedrungenen potentiellen Mörder auf die Frage nach dem Aufenthalt des von ihm verfolgten Opfers wahrheitsgemäß antworten, von Bonhoeffer als eine "groteske Folgerung " und eine zum „frevelhaften Übermut gesteigerte Selbstgerechtigkeit“ gewertet wird; vgl. ders., DBW 6 (Ethik), S. 280, mit Bezug auf Kant, Werke VII, S.637-643 (Über ein vermeintliches Recht).

59 Siehe S. $170 \mathrm{ff}$. 


\section{Die Rechte leiblichen Lebens}

Wir haben bisher Dietrich Bonhoeffers theologisches Verständnis der Krankheit näher beleuchtet, mit dem Ergebnis, daß er das Phänomen der Krankheit von der Nähe des leidenden und gekreuzigten Gottessohnes her qualifiziert. Damit wird die Menschlichkeit Christi - in dessen tröstender Zuwendung zu den Kranken wie in seiner mitleidenden Solidarität - zum Paradigma für den Umgang mit der Krankheit als Teil menschlicher Lebenswirklichkeit, mehr noch: für die göttliche Kraft, die sich in der Ohnmacht verbirgt. An Bonhoeffers in vier Sachmomente gegliedertem Modell einer Verantwortungsethik haben wir, vor allem mit Blick auf die Stichworte „Stellvertretung“ und „Wirklichkeitsgemäßheit“, die Aspekte medizinischer Ethik erweitern können: Verantwortung gegenüber kranken Menschen bedeutet zunächst Akzeptanz der grundlegenden Stellvertretung Gottes für die Menschen, d.h., wer Verantwortung übernimmt, wird zunächst in die Nachfolge eines Größeren gestellt. Insofern ist der Rahmen der beruflichen Verantwortung nicht durch die treue Erfüllung eigengesetzter Berufspflichten zu erreichen, sondern durch die demütige Erkenntnis dessen, daß ein Mensch vor Gottes Maßstäben allemal scheitern wird. Berufliche Verantwortung bedeutet nach Bonhoeffer, dem Ruf Christi, jeweils konkret zu folgen.

Damit haben wir zum Thema „Krankheit“ bzw. „beruflicher Umgang mit der Krankheit" in Bonhoeffers Theologie bereits wesentliche Eckdaten markiert. Jedoch müssen wir für das folgende nun noch einmal neu ansetzen, um die Überlegungen zur „Krankheit“ auf die Fragen der körperlichen und geistigen Behinderung erweitern zu können und damit die Frage unheilbarer Krankheit sowie die damit zusammenhängenden zeitgeschichtlichen Probleme der Sterilisation und "Euthanasie" in den Blick zu nehmen. Bonhoeffer hat sich mit dieser Problematik in der Ethik ausführlich auseinandergesetzt. Die Frage eines „Rechtes auf leibliches Leben“ stellt er in den Kontext der Erörterungen zum „natürlichen Leben“ und damit in den Gesamtzusammenhang vom „Vorletzte[n]“ - dem das „natürliche Leben“ zugeordnet wird - und „Letzte[n]“. Diese geprägten Begriffe werden sich in der nachfolgenden Besprechung weiter erschließen.

Entscheidend ist, daß Bonhoeffer die Frage des „Rechtes auf leibliches Leben“, oder im weiteren Sinne die Frage der Menschenrechte, in zwei weitgreifende $\mathrm{Zu}$ sammenhänge einordnet: in den Zusammenhang von Rechtfertigung und Recht und den von Letztem und Vorletztem. Wie die Rechtfertigung die Kernaussage des „Letzten“, der Christuswirklichkeit, ist ${ }^{60}$, so gehört das Recht in die Wirklichkeit des „Vorletzten“"61. Von den Rechten natürlichen Lebens und den Men-

60 "Ursprung und Wesen alles christlichen Lebens liegen beschlossen in dem einen Geschehen, das die Reformation Rechtfertigung des Sünders aus Gnaden allein genannt hat." DBW 6 (Ethik), S. 137.

61 Wolf, Letztes, verweist darauf, daß ähnliche Überlegungen (ohne diese Terminologie) bereits in den Erwägungen zur theologischen Begründung der Weltbundarbeit (1932) und der "Nachfolge" 
schenrechten zu sprechen, heißt also, das "natürliche Leben“ im „Vorletzten“ näher ins Auge zu fassen.

\section{Das „natürliche Leben"}

Das "natürliche Leben“, verstanden als das leibliche und geistige, im Unterschied zum geistlichen, dem Glaubensleben, wird als das "nach dem Fall auf das Kommen Jesu ausgerichtete" Leben definiert (S.165); das natürliche Leben ist zu unterscheiden vom "Geschöpflichen“, dem Leben vor dem Fall, ebenso wie vom Sündhaften als demjenigen, was das Natürliche vom Geschöpflichen trennt. Das Natürliche kann nach einer formalen Seite durch den Glauben und nach einer materialen Seite durch die Vernunft erkannt werden, worauf später noch näher einzugehen sein wird.

Bonhoeffer verwahrt sich mit dieser Definition gegen ein doppeltes Mißverständnis: daß entweder das Natürliche oft nahezu mit dem Sündigen gleichgesetzt und dadurch entwertet oder umgekehrt die natürlichen Ordnungen überbewertet werden und den "Glanz der Urgeschöpflichkeit" (S.163) erhalten, besonders kraß etwa im Ansbacher Ratschlag von 1934, wo Familie, Volk und Rasse (Blutzusammenhang) als Schöpfungs- und Erhaltungsordnung charakterisiert wurden ${ }^{62}$. Indem Bonhoeffer das Natürliche dem Vorletzten zuordnet, die Rechtfertigung des Sünders aber dem Letzten (S.140), kann er beide als „Wegbereitung und Einzug" aufeinander beziehen, was Titel und Gliederungsprinzip für den geplanten zweiteiligen Gesamtaufbau der "Ethik“ sein sollte ${ }^{63}$. Der Begriff "Wegbereitung" bezeichnet nicht etwa eine dem Menschen mögliche Methode, um kraft eigener Leistung zu Gott zu kommen, sondern "geht im Unterschied zu allen Methoden gerade von der klaren Erkenntnis aus, daß Christus selbst den Weg gehen muß; es ist nicht unser Weg zu ihm, sondern sein Weg zu uns, der bereitet werden soll und der nur in dem Wissen bereitet werden kann, daß er ihn sich selbst bereiten muß" (S.159). Der Sache nach ist dieses Verständnis von Wegbereitung verwandt mit Karl Barths späterer Lehre von den "Gleichnissen des Himmelreiches", wonach immer wieder in der Welt (auch außerhalb der Kirche) Ereignisse geschehen und Gestaltungen menschlichen Zusammenlebens vorkommen, die gleichnishaft vorweg abbilden, was im Reich Gottes zu seiner Erfüllung und Wahrheit kommen wird. Dieser Gleichnischarakter geschichtlicher Ereignisse und Entwürfe ist aber nicht aus sich selbst heraus offenkundig, sondern erschließt sich erst von der Wahrheit des Reiches Gottes her und kommt in der Verkündigung zur Sprache. Bonhoeffers Begriff der „Wegbereitung“ akzentuiert allerdings stärker als Barths „Gleich-

(1937) auftauchen, allerdings damals noch im Rahmen der Ekklesiologie. Die Terminologie geht auf Karl Barth zurück, bei dem sie freilich nicht diesen prominenten Stellenwert besitzt; vgl. Feil, Theologie, S. 298; DBW 9, S.164, Widmann an Bonhoeffer, 29.4. 1926.

62 Vgl. Schmidt, Bekenntnisse 1934, S. 102-104.

${ }^{63}$ Vgl. GS VI, S. 492, Bonhoeffer an Bethge, 27.11. 1940. 
nisse des Himmelsreiches" die Rolle, die hierbei dem menschlichen Handeln zukommt.

Von dieser Prämisse aus kann Bonhoeffer unbefangen alles Menschliche und Gute „für Jesus Christus in Anspruch [nehmen], besonders dort, wo es als unbewußter Rest einer Bindung an das Letzte dasteht" (S. 162). Mit diesem Gedanken vermag Bonhoeffer jene die Kirche beschämende Beobachtung zu verarbeiten, daß immer wieder Menschen ohne Bekenntnis zum christlichen Glauben gleichwohl engagierter für Bildung, Humanität, Freiheit, Toleranz oder Menschenrechte eintreten als manche Christen ${ }^{64}$. Immer bleibt die Zuordnung eindeutig: das Vorletzte ist vom Letzten, das natürliche Leben vom göttlichen Leben her qualifiziert. „Was an Menschlichem und Gutem in der gefallenen Welt gefunden wird, es gehört auf die Seite Jesu Christi." (S. 161)

Angesichts dieses hohen Anspruchs gilt es, das Verhältnis des „Natürlichen“ zur Vernunft näher zu bestimmen. Bonhoeffer differenziert für das „Natürliche“ zwischen einer formalen Bestimmung, die durch den Erhaltungswillen Gottes und die Ausrichtung der Welt auf Christus angesprochen ist, und der inhaltlichen Bestimmung des Natürlichen durch die Vernunft (S.166ff.). Gegen den Anspruch des Naturrechtdenkens wird sofort differenziert: „Die Vernunft ist nicht ein über das Natürliche erhabenes göttliches Erkenntnis- und Ordnungsprinzip im Menschen, sondern sie ist selbst ein Teil dieser erhaltenen Lebensgestalt. . " (S. 167)

Bonhoeffers Gedankengang findet eine Parallele in Luthers „Disputatio de Homine $^{65}$ (1536), dem dichtesten und geschlossensten Entwurf seiner theologischen Anthropologie. Dort qualifiziert Luther die Vernunft theologisch, nicht philosophisch (These 10: „non a priore sed a posteriore“) als Erkenntnismittel für die Di-

${ }^{64}$ Als „unbewußtes Christentum“ taucht dieser Gedanke später wiederholt auf, das erste Mal interessanterweise in einem teilweise autobiographischen Romanfragment, in dem er den Eltern der Hauptfigur zugeschrieben wird, von denen der Vater Arzt, die Mutter Hausfrau ist. Bei diesen habe das unbewußte Christentum bewirkt, daß sie sich nicht vom "Geist des verkehrten Ehrgeizes, des Karrieremachens, der Titel und der Orden" - auch einer Chiffre für die moralische Korruption der Menschen im Nationalsozialismus - hätten anstecken lassen; vgl. Bonhoeffer, Fragmente, S.99. Im Brief an Bethge vom 27.7. 1944 deutet Bonhoeffer an, daß seine Überlegungen zum "unbewußten Christentum“ in Zusammenhang stehen mit seinen frühen Überlegungen zu fides directa und fides reflexa bzw. zu actus purus und actus reflexus; vgl. Bonhoeffer, Widerstand, S. 405; dazu Feil, Theologie, S. 83 ff., $376 \mathrm{f}$.

${ }^{65}$ Luther, Werke Bd. 39 I, S. 174-180. Dazu Stange, Zur Auslegung, S. 306-315; Ebeling, Lutherstudien II; Peters, Mensch, S. 27-59. Bonhoeffer hatte Luthers Disputationen in einer Vorlesung behandelt; vgl. GS V, S.222. Dagegen verweisen die Herausgeber der Ethik-Ausgabe DBW 6 auf Bonhoeffers Vorlesung Schöpfung und Fall und die dort behandelte Unterscheidung Augustins zwischen forma und materia, vgl. DBW 3, S.35ff. Augustin verwendet diese Unterscheidung im Rahmen seiner Schöpfungslehre bezüglich einer stufenweisen Schöpfung von Materie und Gestalt. Bonhoeffer referiert diese Lehre und schließt sich ihr an. Dies alles stellt aber noch keinen Bezug zur hier behandelten Frage der christologischen Erkenntnis des Natürlichen her. Hierzu ist vielmehr Bonhoeffers Christologie-Vorlesung zu vergleichen, in der Christologie als „Logologie" nach einer "formalen" und einer ,inhaltlichen" Seite entfaltet wird. Nach ihrer formalen Seite ist Christologie die "Ermöglichung von Wissenschaft überhaupt", nach ihrer inhaltlichen Seite verweist die Christologie die Vernunft „auf ihre Grenze“; vgl. GS III, S. 172; dazu Claß, Verzweifelter Zugriff, S. 23-39. 
mension des "coram mundo“, nicht nur in bezug auf die Natur, sondern auch auf die zwischenmenschlichen Beziehungen. Die Vernunft ist „im Vergleich mit allen übrigen Dingen das Beste von diesem irdischen Leben und geradezu etwas Göttliches" (These 4). Für die Gotteserkenntnis allerdings ist sie unbrauchbar und weiß nicht von sich aus um ihre Grenzen. Entscheidend ist die 34. These („Daher verhält sich der Mensch dieses Lebens für Gott wie die ,reine Materie‘ [pura materia] gegenüber dem Leben seiner zukünftigen Form [ad futurae formae suae vitam]“), in der Luther das Verhältnis von „forma“ und „materia“" trotz aristotelischer Terminologie nicht eigentlich aristotelisch, sondern eschatologisch bestimmt.

Hingegen unterscheidet sich Bonhoeffers Gebrauch der Begriffe „formal“ und "material“ von dem Emil Brunners ${ }^{66}$ und Robert Jelkes ${ }^{67}$. Beide hatten in den dreißiger Jahren eine vom Sündenfall nicht betroffene „formale" Vernunft von einer vom Sündenfall betroffenen „materialen“ Vernunft abzugrenzen versucht. Dagegen hatte Bonhoeffer sich in einer Rezension mit folgendem Argument gewandt: „Ist nicht gerade dort, wo man von der Verkehrtheit der ganzen (auch der formalen) Vernunft weiß und nun - eben weil man einmal ohne die Vernunft auch theologisch nicht denken kann - dies schartige, mißratene Werkzeug nimmt wie es ist, im vollen Bewußtsein, damit nichts rechtes zustande bringen zu können, ist nicht gerade dort die christliche Bescheidung der Vernunft, die Buße, die die Vernunft tun muß, echter, als dort, wo man sich dieser radikalen Einsicht versagt und immer noch versucht, wenigstens etwas Gutes an diesem schadhaften Mittel ausfindig zu machen, und sich dann auf dies Gute verläßt und sich in ihm gesichert fühlt? Eben weil Barth weiß, daß es mit der ganzen Vernunft nichts ist und daß er sie doch nicht entbehren kann, eben darum kann er ja mutiger und unbefangener, vernunftgemäß reden als einer, der der Vernunft noch ein letztes Recht wahren will“68. Die referierte christologische Bestimmung des Natürlichen beinhaltet also just nicht, wie Bonhoeffer vorgeworfen wurde, eine „Distanzierung von rationaler Handlungskontrolle ${ }^{* 69}$. Vielmehr will Bonhoeffer die Vernunft, im Glauben um ihre Grenzen wissend, instrumental verwenden.

Indem Bonhoeffer das Vorletzte vom Letzten nicht trennt, sondern das „Natürliche“ definiert als die „von Gott der gefallenen Welt erhaltene Gestalt des Lebens, die auf die Rechtfertigung, Erlösung und Erneuerung durch Christus aus-

${ }^{66}$ Brunner, Natur. Bonhoeffer dürfte von dem leidenschaftlichen Protest Barths, Nein!, beeinflußt gewesen sein.

${ }^{67}$ Jelke, Vernunft.

${ }^{68}$ Vgl. GS III, S. 137 (Rex. Jelke, Vernunft).

${ }^{69}$ So Krause, Dietrich Bonhoeffer, S.62. Krause sieht die Schwäche einer christologischen Würdigung des Natürlichen darin, daß Bonhoeffer den Staatsstreich nicht, wie Luther und die Scholastik, auf die Liebe und die Vernunft gegründet habe. Formal betrachtet kann diese Kritik Bonhoeffers christologische Bestimmung des Natürlichen schon deshalb nicht treffen, weil der Staatsstreich dort überhaupt nicht behandelt wurde. Krause scheint sich mehr auf seinen eigenen Disput mit Bonhoeffer zu beziehen. Seinerzeit störte er sich an dem hohen Maß an Verbindlichkeit, mit der Bonhoeffer den "Widerstand" gegen eine "tyrannische Obrigkeit" einforderte; vgl. GS VI, S.441, Krause an Bonhoeffer, 18.2. 1939. 
gerichtet ist“ (S.166), kann er dem natürlichen menschlichen Leben eine „Wür-

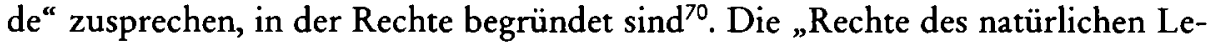
bens" versteht er als „Abglanz der Schöpfungsherrlichkeit Gottes mitten in der geschaffenen Welt" (S. 174). Da er sein Verständnis des Natürlichen vom Evangelium und nicht vom Gesetz her gewinnt, ordnet er die Rechte natürlichen Lebens als allen ethischen Pflichten höherrangig vor. Mit der Anerkennung der Rechte natürlichen Lebens wird „nicht das Geschöpf, sondern der Schöpfer" geehrt, der „Reichtum seiner Gaben“" anerkannt (S. 173).

Bonhoeffers Verständnis der Menschenwürde dürfte sich der Auseinandersetzung mit Immanuel Kant verdanken, wie der in diesem Zusammenhang von Bonhoeffer verwendete Begriff der „Selbstzwecklichkeit“ zeigt ${ }^{71}$. Allerdings sieht Bonhoeffer im Unterschied zu Kant die Menschenwürde nicht in der Vernunft und im kategorischen Imperativ begründet, sondern in Gottes gnädiger Zuwendung zum Menschen. Dies hat Konsequenzen für die Würde derjenigen Menschen, denen die Vernunft fehlt oder zu fehlen scheint: Weil Gott sich auch und gerade geistig Behinderten zuwendet, kann ihnen die Menschenwürde nicht abgesprochen werden ${ }^{72}$. Dabei kann Bonhoeffer auf eine bis auf Luther zurückgehende theologische Tradition zurückgreifen, derzufolge der Mensch nicht ${ }^{73}$ durch seine Vernunft definiert werden kann („Homo ex homine definiri nequit $\left.{ }^{\text {“ }}\right)^{74}$, sondern dadurch, daß er aus Glauben gerechtfertigt wird. Weder der Glaube noch das Rechtfertigungsgeschehen setzt, Bonhoeffer zufolge, das empirische Vorhandensein der Vernunft voraus. Damit kann das Rechtfertigungsgeschehen in einem strikten Sinne voraussetzungslos gedacht werden: Um aus Gnade im Glauben gerechtfertigt zu werden, bedarf es der Vernunft nicht, kann man auch schwer geistig behindert $\operatorname{sein}^{75}$.

${ }^{70}$ Zur Begriffsgeschichte vgl. Panajotis, Würde.

71 „Im Reich der Zwecke hat alles entweder einen Preis, oder eine Würde. Was einen Preis hat, an dessen Stelle kann auch etwas anderes als Äquivalent gesetzt werden; was dagegen über allen Preis erhaben ist, mithin kein Äquivalent verstattet, das hat eine Würde.“ Kant, Werke Bd.6 (Grundlegung zur Metaphysik der Sitten), S.68. Vgl. damit Bonhoeffer Auffassung: „Das leibliche Leben ist wie das Leben überhaupt sowohl Mittel zum Zweck wie Selbstzweck." DBW 6, S. 179.

72 Zwar hat nun Kant eine derartige Konsequenz nicht gezogen (anders: Hegselmann/Merkel, Debatte, S. 12), doch da er die Gültigkeit des kategorischen Imperativs bei allen Vernunftwesen unabhängig von ihrer menschlichen Natur behauptete, mußte er sie auch bei denjenigen Vernunftwesen postulieren, denen ihrer menschlichen Natur nach die Vernunft zu fehlen scheint, den "Irren“. Kant zog daraus die eigenartige Konsequenz, zwar das „Irrereden“ bei Fieber als körperliche Krankheit zur Behebung den Ärzten, das eigentliche „Irresein" aber der Philosophie und nicht der Medizin als Aufgabe zu überlassen; vgl. Kant, Werke Bd.10 (Anthropologie in pragmatischer Absicht), S. 529; dazu Dörner, Bürger, S.200-212.

${ }^{73}$ Vgl. aber die Definition des Menschen als „animal rationale“ bei Aristoteles, Eth. Nic. 1177 b. Diese Definition war schon bei Aristoteles mit der Ausgrenzung derjenigen verbunden, die keine Vernunft besitzen: Behinderte Kinder dürfen ausgesetzt werden. Siehe S.24.

${ }^{74}$ Zum Problem der Definierbarkeit des Menschen vgl. Jüngel, Gott, S. $347 \mathrm{ff}$.

75 Zur Frage der "fides directa" in der Taufe bei "unmündige[n] Kindern, Idioten" vgl. einerseits DBW 1 (Sanctorum Communio), S.71; andererseits: DBW 2 (Akt und Sein), S. 159; GS III, S. 441 (Taufgutachten); DBW 9 (Luthers Anschauung vom Heiligen Geist), S.396f. Die gänzlich andere 


\section{Der rechtstheologische Ansatz}

Generell hat Bonhoeffer mit der Behandlung von Menschenrechtsfragen im Rahmen einer theologischen Ethik ebenso wie auch hinsichtlich der materialethischen Überlegungen in diesem Zusammenhang Neuland betreten. In Deutschland standen vor 1945 die beiden großen Kirchen der Menschenrechtsbewegung eher kritisch, wenn nicht sogar ablehnend gegenüber, weil man sie mit kirchenfeindlichen Tendenzen in der Französischen Revolution in Verbindung brach$\mathrm{te}^{76}$. Die Grundrechte, wie sie im Verfassungsentwurf der Paulskirche von 1848 und in der Weimarer Reichsverfassung von 1919 ihren Niederschlag gefunden haben, wurden als solche in theologischen Ethiken nicht behandelt. Bonhoeffer hat sich bezeichnenderweise das erste Mal in den USA mit den Menschenrechten beschäftigt, wo er am Union Theological Seminary in einem Seminar zum Thema "Church and Community" bei C.C. Webber die American Civil Liberty's Union kennenlernte, die für die Einhaltung der Menschenrechte und besonders die Gleichberechtigung der Schwarzen eintrat. Hieran knüpfte er an, als er eine Woche nach der "Machtergreifung" im Anschluß an die von ihm ironisch „Exorzismus“ genannten Kommunistenverfolgungen die Gründung einer deutschen Civil Liberty's Union forderte 77.

Die Unterscheidung zwischen Letztem und Vorletztem, zwischen Wegbereitung und Einzug, ermöglicht es Bonhoeffer, die Rechte natürlichen Lebens weder naturrechtlich noch rechtspositivistisch bestimmen zu müssen. Eigenschaft des Rechtes ist, zwischen den Menschen und nicht vor Gott zu gelten: „Recht gibt es nicht vor Gott, sondern das rein gegebene Natürliche wird dem Menschen gegenüber zu Rechten." (S. 173) Das Recht ist nicht in erster Linie etwas, was "der Mensch in seinem eigenen Interesse einklagen kann“ (S.174). Die Rechte natürlichen Lebens haben also selbst dort Gültigkeit, wo man sie vor Gericht nicht einklagen kann, wie es im Nationalsozialismus in vieler Hinsicht der Fall war. Da das Recht für Bonhoeffer in den Bereich des Vorletzten gehört, versteht er das Eintreten für die Menschenrechte als "Wegbereitung“.

Bonhoeffers These vom "Letzten und Vorletzten" korrespondiert, in der theologischen Grundlegung wie in der daraus zu folgernden Rechtstheorie, in vieler-

Argumentation Emil Brunners ist hier zu vergleichen. Brunner sieht im Personsein des Menschen über den Weg einer analogia entis einen "Anknüpfungspunkt" für die Verkündigung und definiert gleichzeitig den Begriff der Person über seine Merkmale, nämlich Selbstbewußtsein, Vernunft und Subjekthaftigkeit. Daraus folgt, daß "Idioten“ als "Nichtpersonen“ oder bestenfalls als „Grenz$\mathrm{fall}^{\circ}$ zu bewerten sind und ihr Glaube zumindest fragwürdig erscheint: „Der Geist [...] fundiert das Personsein. Man braucht kein großer Geist zu sein, um ein wahrhaft glaubender und liebender zu sein; aber obne Geist, als Idiot kann man auch nicht glauben." Brunner, Mensch, S. 351. Ähnlich Gerstenmaier: „Ist der Idiot wirklich Person? Das Fehlen personhafter Aktualität, die Nichtfeststellbarkeit der Urphänomene des Sittlichen spricht dagegen. Er ist offensichtlich kein Subjekt, sondern Objekt. " Ders., Xirche, S. $179 f$.

76 Belege bei Huber/Todt, Menschenrechte, S. 45-55.

"V VI. GS VI, S. 260, Bonhoeffer an Niebuhr, 6.2. 1933; Bethge, Bonhoeffer, S.200f. 
lei Hinsicht mit der Barthschen Gegenüberstellung von Rechtfertigung und Recht, also jener politisch besonders umstrittenen Schrift, in der Barth für die Demokratie, für den Sturz des NS-Regimes und für die Landesverteidigung der Deutschland benachbarten Staaten eintrat. Von grundlegender Bedeutung wurde für Bonhoeffer Barths These, daß Recht analogisch von der in Christus vollzogenen Versöhnung und nicht von der Schöpfung oder vom Gesetz her zu denken sei. Barth geht von einer fundamentalen Differenz zwischen Kirche und Staat aus, sieht aber zugleich eine „Beziehung zwischen der von Gott in Jesus Christus ein für allemal vollzogenen Rechtfertigung des Sünders allein durch den Glauben und dem Problem des menschlichen Rechtes ${ }^{\text {c78 }}$. Eine naturrechtliche Rechtsbegründung ist damit ausgeschlossen.

In seinem Manuskript „Kirche und Staat" nimmt Bonhoeffer Barths Überlegungen sehr weitgehend auf ${ }^{79}$. Für Bonhoeffers rechtstheoretische Überlegungen sind weiterhin noch seine juristischen Gesprächspartner und Schwäger Gerhard Leibholz, der spätere Verfassungsrichter ${ }^{80}$, und Rüdiger Schleicher, Ministerialrat im Reichsluftfahrtministerium (1945 wegen Teilnahme am Widerstand ermordet) ${ }^{81}$, zu nennen. Mit dem Erstgenannten diskutierte Bonhoeffer auch Barths Schrift und entwickelte dabei dialektisch seinen eigenen Ansatz: Hinsichtlich der gemeinsam interessierenden Frage des Naturrechts („unser altes Diskussionsthema") hält Bonhoeffer die Theorie, derzufolge es in der Schöpfung Rechtsprinzipien gebe, „die als absolut gültige angesehen werden können“ (= lex naturae), für ebenso fragwürdig wie ihr Gegenteil, daß Recht allein „an faktische Macht" gebunden sei (= Rechtspositisvismus). Sowohl eine Relativierung der Offenbarung (im Naturrecht) als auch eine Relativierung des Geschichtlichen und der Schöpfungsnormen (bei „streng reformatorischer" Systematik, aber auch beim Rechtspositivismus) sei zu vermeiden: „Karl [Barth] hat ja nun den Versuch gemacht, auf Grund der streng reformatorischen These dennoch die Relati-

78 Vgl. Barth, Rechtfertigung, S. 40-43; dazu Reuter, Rechtsbegriffe, S. 202-215.

${ }^{79}$ Man vergleiche etwa die ungewöhnliche Pilatus-Exegese (Barth, Rechtfertigung, S. 9-14, mit Bonhoeffer, Ethik ${ }^{11}$, S.358f.), die Übersetzung von "exousiai" mit „Engelmächte“ (Barth, S. 15, Bonhoeffer, S.353), das Verständnis des Staates vom himmlischen Jerusalem her (Barth, S. 22, Bonhoeffer, S. 359), das Verständnis des apokalyptischen Staates Apk, 13 als defizientem Modus des Staates von Römer 13 (Barth, S.16, Bonhoeffer - variierend - S. 365), die Aufgabendefinition des Staates nach 1. Tim. 2, 2 (Barth, S. 19, Bonhoeffer S. 368), die Stellungnahme zu Steuer, Eid und - bei Bonhoeffer entgegen seiner eigenen Position - zum Kriegsdienst (Barth, S. 42, Bonhoeffer, S. 365) usw. Was es für die Interpretation dieses oft als undemokratisch und autoritär angesehenen Textes bedeutet, daß er aus einer Schrift Barths stammt, in der Barth eindeutig für die Demokratie plädierte, soll hier nicht diskutiert werden.

${ }^{80}$ Zur Rezeption von Leibholz, Gleichheit, bei Bonhoeffer vgl. Strohm, Theologische Ethik, S. $97 \mathrm{ff}$., $122 \mathrm{ff}$. Die meisten Anspielungen auf die Gleichheitsschrift von Leibholz in Bonhoeffers Ethik finden sich im Kapitel „Suum cuique“, vgl. DBW 6 (Ethik), S.174-179. Kritisch zum Gleichheitsgedanken von Leibholz und seiner Durchsetzung in der Rechtsprechung des Verfassungsgerichtes Perels, Gleichheitssatz.

${ }^{81}$ Schleicher beschäftigte sich im Anschluß an den niederländischen Rechtsphilosophen Hugo Grotius intensiv mit der Frage einer naturrechtlichen Begründung der Menschenrechte und ließ Bonhoeffer an diesen Überlegungen teilhaben; vgl. Gerrens, Staatsdienst. 
vierung des Geschichtlichen zu vermeiden. Das ist sehr bestechend. Er bezieht (gut biblisch) alle Ordnungen der geschaffenen Welt streng auf Christus und sagt, daß sie nur von ihm her recht zu verstehen seien und an ihm ihre Ausrichtung finden müßten. Das muß man lesen. Auf die Behandlung dieser Probleme muß dann m.E. die Frage nach dem Verhältnis von Recht, Gerechtigkeit und Liebe (im Sinne der Bergpredigt) folgen. Schließen sie sich aus? Gehören sie (obwohl in Antithese!) doch zusammen? (das würde ich meinen). Recht, das auf Gerechtigkeit ausgerichtet ist und durch Macht eine geschichtliche Wirklichkeit (und keine abstrakte Idee!) ist, ist der ,Pädagoge auf Christus hin', wie es im Galaterbrief K.3,24 heißt. Obwohl Liebe inhaltlich, wie Brunner gut ausführt, sich vom Recht wesenhaft unterscheidet (Preisgabe des eigenen Rechtes um Gottes und des Nächsten willen), kann sie nur innerhalb einer Rechtsordnung praktiziert werden Es scheint mir nun letzter Sinn allen Rechtes auf Erden zu sein, trotz aller durch Macht durchzusetzenden Strenge, die Möglichkeit der Liebe im Christentum zu gewährleisten, ohne je mit ihr identisch zu werden. Recht ist also ebenso antithetisch zur Liebe wie notwendig um ihrer willen" ${ }^{82}$.

Damit lehnt Bonhoeffer das in einer Theologie neulutherischer Prägung verbreitete Nebeneinander von Schöpfungsordnung und Erlösungsordnung, von Welt und Reich Gottes, von äußerlichem Charakter der Rechtsordnung und innerlichem Wesen der Liebe, ab. Bonhoeffers dialektische Aussage (Recht und Liebe gehören ,in Antithese [...] zusammen") signalisiert, daß er umgekehrt auch eine bloße Identifikation von Liebe und Recht verwirft. Eine lieblose Rechtsordnung und eine rechtlose Liebesordnung erscheinen ihm gleichermaßen wenig erstrebenswert ${ }^{83}$. Liebe, besonders Feindesliebe, überschreitet nach Bonhoeffer jede Rechtsforderung, kann nach der Bergpredigt einseitig ausgerichtet sein und die Preisgabe eigenen Rechtes beinhalten. Recht dagegen ist auf Wechselseitigkeit ausgerichtet, regelt miteinander konkurrierende Rechtspositionen und ist in der Sozialität des Menschen begründet, die in der Liebe zur Erfüllung kommt. Recht und Liebe bleiben in unauflöslicher Spannung aufeinander bezogen.

Bonhoeffer weiß in seiner Darlegung der Menschenrechte auch durchaus positiv an die klassischen Menschenrechtserklärungen des achtzehnten Jahrhunderts anzuknüpfen. In historischer Hinsicht schließt er sich der von dem Juristen Georg Jellinek aufgestellten, von Max Weber, Ernst Troeltsch und Karl Jaspers verbreiteten These an, derzufolge die amerikanische Revolution im Unterschied zur Französischen in ihrer Menschenrechtsproklamation stark durch christlichspiritualistische Impulse bestimmt worden $\mathrm{se}^{84}$. Dennoch bedarf es dieses historischen Nachweises für die systematische Begründung nicht, da Bonhoeffer aufgrund seines Verständnisses von Wegbereitung alles Menschliche und Gute für Christus in Anspruch nehmen kann.

82 GS III, S. 34-36, Bonhoeffer an Leibholz, 7.3. 1940; dazu vgl. Pangritz, Barth, S.71 f.

${ }^{83}$ So ohne Bezugnahme auf Bonhoeffer die Formulierung von Huber, Recht, S. 249.

${ }^{84} \mathrm{Vgl}$. DBW 6 (Ethik), S.116f. Anm. 94. 
Wie die klassischen Erklärungen die Menschenrechte als Rechte des einzelnen formulieren, kann auch Bonhoeffer sagen: „Daß es ein natürliches Recht des Einzelnen gibt, folgt aus dem Willen Gottes, den Einzelnen zu schaffen und ihm das ewige Leben zu schenken." (S.177) Und ebenso wie jene Erklärungen des achtzehnten Jahrhunderts die Menschenrechte als „angeborene“ Rechte bezeichnen, beschreibt auch Bonhoeffer das wichtigste Recht natürlichen Lebens, das Recht auf leibliches Leben, folgendermaßen: „Es ist dies nicht ein Recht, das wir uns geraubt oder erworben hätten, sondern es ist im eigentlichen Sinne ,mit uns geborenes', empfangenes Recht, das vor unserem Willen da ist, das im Seienden selbst ruht. Da es nach Gottes Willen menschliches Leben auf Erden nur als leibliches Leben gibt, hat der Leib um des ganzen Menschen willen das Recht auf Erhaltung. Da mit dem Tode alle Rechte erlöschen, so ist die Erhaltung des leiblichen Lebens die Grundlage aller natürlichen Rechte." (S. 179)

Dennoch besteht ein wichtiger Unterschied zwischen Bonhoeffers Definition der Rechte natürlichen Lebens und den vorherrschenden liberalen Menschenrechtsbegründungen. Ihnen zufolge ist das "Individuum“ von Geburt an Träger aller Rechte und tritt gemäß der Theorie des Gesellschaftsvertrages einzelne dieser Rechte an das Gemeinwesen ab, das seinerseits zum Schutz dieser Individualrechte verpflichtet is ${ }^{85}$. Für Bonhoeffer dagegen ist das "natürliche Leben “ und nicht das Individuum primär der Rechtsträger. Ihm geht es nicht etwa darum, das Kollektiv, die Gemeinschaft, die Gesellschaft oder den Staat dem Einzelnen überzuordnen mit der Folge, daß die Rechte des einzelnen im Recht des Kollektivs aufgehen würden ${ }^{86}$. Für ihn kommt es vielmehr darauf an, seiner alten These von der Gleichursprünglichkeit von Individualität und Sozialität ${ }^{87}$ auch in der Rechtstheologie Rechnung zu tragen: „Das Recht des Einzelnen ist die tragende Kraft des Rechtes der Gemeinschaft wie umgekehrt die Gemeinschaft das Recht des Einzelnen schützt. " (S. 177) Somit sieht er in den Freiheitsrechten des einzelnen nur einen bestimmten Aspekt, nicht aber eine erschöpfende Behandlung der Rechte natürlichen Lebens.

${ }^{85}$ Vgl. Tödt, Bonhoeffers theologische Ethik, S.141f.

${ }^{86}$ So Kodalle, der bei Bonhoeffer einen "fatale[n] Vorrang des Kollektiven" wirksam sieht; vgl. ders., Bonhoeffer, S.16, 35. Kodalles Maßstab ist sein eigenes Verständnis des Christlichen, welches "nicht sein eigener Inhalt, sondern eine bestimmte Intensitätsform der individuellen Selbstwerdung" sei; vgl. ebenda, S.33. Dabei vernachlässigt er, daß im christlichen Verständnis das „Individuum" fundamental mit seinem "Nächsten" verbunden ist.

${ }^{87}$ So - im Rahmen der Ekklesiologie - schon DBW 1 (Sanctorum Communio), S.34. Graf konstatiert hier zu Recht eine „Verabschiedung der neuzeitlichen, cartesianisch-idealistischen Subjektsphilosophie", versteigt sich aber zu der Aussage, dies beinhalte eine ninnere Gleichschaltung", und läßt das in dem Vorwurf gipfeln, Bonhoeffers "Theorie der Kirche" drohe "in ein potentiell totalitäres Sozialkonzept umzuschlagen“. Vgl. ders., Innerlichkeit, S.388. Grafs Kategorie der "inneren Gleichschaltung “ verschleiert den historischen Tatbestand, daß Bonhoeffer die „äußere $^{\star}$, die nationalsozialistische "Gleichschaltung" der Kirche ablehnte. Ihr liegt die fragwürdige Methodik zugrunde, in einem ersten Schritt - wohlbemerkt im Namen des Pluralismus - die neuzeitliche, cartesianisch-idealistische Subjektsphilosophie für die einzige im Pluralismus mögliche Denkungsart zu erklären, um dann in einem zweiten Schritt Abweichungen davon als antipluralistisch zu brandmarken. 


\section{Die materialethische Konkretion}

An den konkreten Ausführungen über die natürlichen Rechte leiblichen Lebens hat Bonhoeffer während seines Aufenthaltes im Kloster Ettal vom November 1940 bis Februar 1941 gearbeitet. Von den vier hier behandelten Themenkomplexen, „Recht auf das leibliche Leben“, „Selbstmord“, „Fortpflanzung und werdendes Leben" und „Freiheit des leiblichen Lebens", sind im Rahmen dieser Arbeit besonders die ersten drei von Interesse. Geplant war eine Fortsetzung „Die natürlichen Rechte geistigen Lebens", von der aber nur Zettelnotizen vorliegen. Hiervon wären für unseren Kontext die Kapitel „Wissen“, „Wissenschaft" und „Bildung" relevant gewesen, in denen Bonhoeffer unter dem Stichwort "geistige Tötung Minderwertiger" und "geistige Vergewaltigung" die propagandistischen Begleitmaßnahmen der „Euthanasie“-Aktion behandeln wollte ${ }^{88}$.

Bonhoeffer gibt keine nähere Begründung für die Auswahl der konkreten Rechte, die er behandelte bzw. zu behandeln plante. Wenn eine Methode bei der Themenauswahl zu erkennen ist, so ist es die, bei der Formulierung der Rechte leiblichen und geistigen Lebens faktische oder gedanklich antizipierte Verletzungen der Menschenwürde zu negieren. Damit können Rechte auf ihren Sinngehalt und ihre etwaigen Konsequenzen hin geprüft, nicht aber hervorgebracht werden. Auf den ersten Blick scheint dies eine sehr begrenzte und damit fragwürdige Vorgehensweise zu sein. Allerdings bieten andere Diskussionsversuche ein ähnliches Bild: So beobachtet ein Grundgesetzkommentator, daß nach 1945 im Parlamentarischen Rat inhaltlich über Menschenwürde nur „negativ im Hinblick auf die Würdeverletzungen durch die nationalsozialistische Gewaltanwendung gesprochen" wurde ${ }^{89}$. Ähnlich resümiert Habermas, in den Grundrechtsteilen der verschiedenen Länderverfassungen sowie in dem nicht in Kraft getretenen Entwurf für eine Verfassung der Deutschen Demokratischen Republik vom April 1990 schwinge das "Echo eines erlittenen Unrechts" mit, das gleichsam „Wort für Wort negiert" werde ${ }^{90}$. Offenbar werden auch in der allgemeinen Verfassungsdiskussion Grundrechte vor allem so definiert, daß konkrete Verletzungen der Menschenwürde ausgeschlossen werden können.

Einen besonderen Akzent setzt Bonhoeffer insofern, als er stärker als die klassischen Menschenrechtstheorien die Leiblichkeit menschlichen Lebens betont. Der Mensch ist und bleibt kraft der Auferstehung des Fleisches leibliches Leben. Der Leiblichkeit als gottgewollter Existenzform kommt die "Würde“ und das Recht der "Selbstzwecklichkeit" zu, die sich in den Freuden des Leibes ausdrückt und willkürliche Schädigung, Folter und Tötung ausschließt. Es umfaßt alle mit dem leiblichen Leben zusammenhängenden Probleme des Wohnens, der

\footnotetext{
${ }^{88}$ Vgl. Bonhoeffer, Zettelnotizen, S.75f., Zettel Nr.49, 51.

89 Vgl. Podlech, in: Azzola, Kommentar, zu Art.1 Abs. 1 Rz. 12, S. 205.

90 Vgl. Habermas, Faktizität, S. 469.
} 
Ernährung, der Erholung, des Spieles und der Geschlechtlichkeit. (S.181) Bonhoeffer zitiert zur Begründung des „Rechtes auf leibliche Freuden“ aus der Weisheit Salomonis. Vielleicht hat er sich dabei auch durch die amerikanische Unabhängigkeitserklärung inspirieren lassen, die in Art. 2 als angeborenes Recht aller Menschen "the pursuit of happiness" nennt

Von der platonischen Auffassung des Leibes als dem Gefängnis der Seele grenzt Bonhoeffer sich explizit ab. (S.180) Doch ergibt sich mit der starken Betonung des Leibes auch ein Unterschied zu Immanuel Kant: Nach Kant kommt allein dem sittlichen Willen, also dem Menschen als Vernunftwesen, nicht aber dem menschlichen Leib eine Würde zu. Kant sieht die Menschenwürde im Sittengesetz begründet und denkt das Sittengesetz von allen empirischen (materialen) Bestimmungsgründen unabhängig, wobei die gesetzgebende Form der Maximen allein den zureichenden Bestimmungsgrund abgibt. Da die empirischen Bestimmungsgründe Begebenheiten in der Natur und folglich dem Gesetz der Kausalität unterworfen sind, ist der sittliche Wille vom Kausalitätsgesetz vollkommen unabhängig. Der menschliche Leib hingegen ist vom Kausalitätsgesetz abhängig, weshalb ihm auch keine Würde zukommen kann. Von der Erhaltung leiblichen Lebens kann Kant nur noch so sprechen, daß er sie als Pflicht des Menschen sich selbst gegenüber kennt, nämlich als Pflicht des Vernunftmenschen gegenüber der eigenen Natur, sich angemessen zu ernähren, sich nicht umzubringen oder zu verstümmeln usw ${ }^{92}$. In diesem Zusammenhang das Streben nach Glück zu denken, ist praktisch unmöglich. Im Unterschied dazu kommt Bonhoeffer zufolge dem menschlichen Leib eine eigene, sogar eine „höhere Würde“ (S.180) zu als dem Geist, was im folgenden zu behandelnde Konsequenzen für Bonhoeffers Darstellung der Rechte leiblichen Lebens hat.

\section{Das „Recht auf Bewabrung leiblichen Lebens" im Widerspruch zur „Euthanasie“}

In einem Brief aus Kloster Ettal schreibt Bonhoeffer: „In meiner Arbeit bin ich jetzt bei der Frage der Euthanasie angekommen. Je mehr ich zum Schreiben komme, desto mehr reizt mich der Stoff. Ich finde die katholischen Ethiken in vieler Hinsicht sehr lehrreich und praktischer als die unseren. Bisher hat man ihnen das immer als ,Kasuistik' angekreidet, heute ist man dankbar für vieles; gerade auch zu meinem augenblicklichen Thema ${ }^{493}$.

Ausgangspunkt von Bonhoeffers Überlegungen ist das „Recht auf Bewahrung des leiblichen Lebens vor willkürlicher Tötung". „Willkürlich“ ist "vorsätzliche" Tötung "unschuldigen" Lebens. (S. 183) Als solche behandelt er auch die vorsätzliche Tötung von Zivilisten, Verwundeten und Kriegsgefangenen. In der

\footnotetext{
91 Vgl. Heidelmeyer, Menschenrechte, S. 54.

92 Vgl. Kant, Werke 7, S. 553-572 (Metaphysik der Sitten).

${ }^{93}$ GS II, S. 394, Bonhoeffer an Bethge, 20.1. 1941.
} 
Todesstrafe oder der Tötung eines feindlichen Soldaten sieht er dagegen nicht die Tötung eines „Unschuldigen" ${ }^{\text {" } 94}$.

Die durch die "Euthanasie“-Aktion akut gewordene Problematik faßt Bonhoeffer in der Frage zusammen, ob unschuldiges Leben, das als nicht mehr lebenswert angesehen wird, auf schmerzlose Weise vernichtet werden darf. (S. 184) Da es sich eindeutig um die vorsätzliche Tötung unschuldiger Menschen handelt, ist klar, daß Bonhoeffer die Frage verneint.

In seiner Argumentation geht er dabei methodisch von dem Prinzip aus, daß eine Tötung nie mit einer Vielzahl von Gründen gerechtfertigt werden kann. „Das Leben darf alle Gründe für sich geltend machen, für die Tötung gilt nur ein einziger Grund “. (S. 185) Unzulässig ist also, eine für sich allein unzureichende Begründung mit Hilfe einer zusätzlichen stichhaltig zu machen, beispielsweise die „Euthanasie“-Aktion kumulativ mit Rücksicht sowohl auf die Kranken als auch auf die Gesunden rechtfertigen zu wollen. Genau dies geschah aber, beispielsweise in dem nur aus propagandistischen Gründen nicht verabschiedeten „Euthanasie“-Gesetz, demzufolge alle diejenigen umzubringen waren, die an einer „unheilbaren, sich oder andere stark belästigenden oder sicher zum Tode führenden Krankheit" leiden".

Anhänger der These, die "Rücksicht auf den unheilbar Kranken“ mache die Tötung erforderlich (S.186), müßten davon ausgehen, daß der Kranke den Tod wünsche oder zumindest in ihn einwillige. Niemand darf sich anmaßen, in bezug auf einen Kranken, etwa einen „Idioten“, der nicht in der Lage ist, einen solchen Wunsch verbindlich zu äußern, einen Todeswunsch zu unterstellen. „Wer aber vermag zu ermessen, wie stark selbst der unheilbar Geisteskranke trotz seines Leides am Leben hängt und wieviel Glücksgefühl er selbst seinem armseligen Leben noch abringt? Es spricht sogar vieles dafür, daß hier die Lebensbejahung besonders stark und hemmungslos ist". (S. 186) Es bleibt lediglich der Fall einer Tötung auf wirklichen Wunsch hin, was Bonhoeffer entweder als "Selbstmord“ oder als diesbezügliche "Beihilfe" behandelt. (S.186f.)

Das Recht auf Selbsttötung wegen schwerer Krankheit bildete bei bewußter Vermischung aller ethischen Kategorien einen Schwerpunkt der NS-Propaganda. So wurde in dem NS-Propaganda-Film „Ich klage an" eine an multipler Skle-

${ }^{94}$ Man kann darin eine Rechtfertigung der Todesstrafe und der Tötung feindlicher Soldaten im Kriege sehen, so Müller, Kirche, S.522 Anm. 939. Diese Interpretation vermag schwer zu erklären, warum Bonhoeffer selbst die aktive Teilnahme an einem Angriffskrieg nicht mit seinem Gewissen vereinbaren konnte; vgl. Bethge, Weg. Sinnvoller erscheint mir ein Vergleich mit dem Völkerrecht, welches die Todesstrafe und die Tötung feindlicher Soldaten im Krieg weder verbot noch gebot, sondern das dem nationalen Recht überließ. Bonhoeffers Theorie der Rechte natürlichen Lebens kann so verstanden werden, daf er unterschied $x$ wischen einem Minimalbestand von Rechten natürlichen Lebens, vergleichbar etwa unseren Grundrechten, und einem positiven Recht, welches den Rechten des natürlichen Lebens nachgeordnet werden muß, stärker historischem Wandel unterliegt, aber deswegen nicht gleichgültig ist. Dieser Interpretation zufolge darf die Gemeinschaft ihre natürlichen Rechte durch Todesstrafe oder Verteidigungskrieg verteidigen, muß es aber nicht tun.

${ }^{95}$ Aly/Roth, Gesetz (Kursivierung U.G.). Ähnlich schon Binding/Hoche, Freigabe. 
rose Erkrankte gezeigt, die von ihrem liebenden Ehemann, einem Professor der Medizin, ein Fläschchen Gift erhielt, um nicht weiter leiden zu müssen ${ }^{96}$. Doch wurde das Recht auf Selbsttötung in seriöser Form auch von Vertretern individualistischer Freiheitsrechte wie der englischen „right-to-death“-Bewegung gefordert $^{97}$. Für Bonhoeffers ethische Überlegung ist die Grundentscheidung maßgeblich, einer durch Krankheit motivierten Selbsttötung keine Sonderrolle bei der ethischen Beurteilung des Selbstmordes einzuräumen. Sofern jemand allein einen Selbstmord begeht, handelt es sich nicht um eine Rechtsfrage, da Recht nur zwischen mehreren Menschen „glaubwürdig“ werden kann. (S.195) Das rechtliche Verbot des Selbstmord(versuchs) in England ist nutzlos. (S.195) „Wer nicht mehr leben kann, dem hilft auch der Befehl, daß er leben soll, nicht weiter, sondern allein ein neuer Geist." (S.181) Dieser Geist kann im Gebet und durch Gottes Gnade gewonnen werden, nicht aber durch Moral oder Gesetz.

Dagegen handelt es sich sehr wohl um eine Rechtsfrage, wenn Dritte an einem Selbstmord beteiligt sind. Der von Bonhoeffer in diesem Kontext verwandte Begriff der „Beihilfe zum Selbstmord“ erinnert an die juristische Terminologie: „Tötung auf Wunsch“ war nach $\$ 216$ Strafgesetzbuch untersagt; „Beihilfe zum Selbstmord“ wurde allenfalls als unterlassene Hilfeleistung nach $\$ 330$ c Strafgesetzbuch verfolgt. Bonhoeffer hat diese Rechtslage nicht kritisiert, hielt freilich die Beihilfe zum Selbstmord, obwohl u. U. straffrei, in der Regel für sittlich unerlaubt. Sofern ein schwer Depressiver den eigenen Tod begehre, müsse der Arzt berücksichtigen, daß es sich um die Bitte eines Kranken handele, der über sich selbst nicht Herr sei. Wenn ein Schwerkranker mit klaren Sinnen den eigenen Tod begehre, sei der Arzt „nicht nur dem Willen“, sondern „auch dem Leben“ des Kranken verpflichtet. (S. 186) Hier zeigt sich die Folge von Bonhoeffers Ausgangsüberlegung, den Rechten natürlichen Lebens - gerichtet auf Lebenserhaltung - gegenüber den Freiheitsrechten des Individuums den Vorrang einzuräumen. Damit scheidet Rücksichtnahme auf den Kranken als sittlicher Rechtfertigungsgrund für eine Tötung aus.

Es fragt sich aber, ob die ärztliche Pflicht dem Leben gegenüber eine unbedingte ist, Leben also um jeden Preis erhalten werden muß. Offenbar im Anschluß an katholische Ethikentwürfe ${ }^{98}$ behauptet Bonhoeffer einen qualitativen

96 Vgl. Roth, ,Ich klage an“.

97 Ehrhardt, Euthanasie, S. 5 f. Ein sittliches Recht auf Suizid will begründen Kamlah, Philosophische Anthropologie, S.174-182.

98 Die Unterscheidung zwischen Töten und Sterbenlassen ist ein klassischer Topos katholischer Moraltheologie, vgl. z. B. Walter, Euthanasie, S.335f.: „Der kategorische Imperativ unbedingter Lebenserhaltung ist eine unhaltbare Fiktion, ein Popanz, gegen den von den Euthanatisten immer wieder ein Windmühlengefecht unternommen wird.“ Ebenda, S.338: „Auch wer die Euthanasie verwirft, wird aus Rücksicht auf die ohnehin schmerzliche Lage des Sterbenden, auf sein Bedürfnis nach Ruhe und Schonung, alle unnützen, die letzten Kräfte zwecklos verzehrenden oder den Kranken aufregenden Pflege- und Heilversuche unterlassen. Die Pflicht der Lebenserhaltung darf nicht zu nutzloser Quälerei verleiten. Die Pflicht des Arztes zur Lebensverlängerung hat ihre in der Natur der Sache liegende Grenze und hört dort auf, wo er außerstande ist das fliehende Leben aufzuhalten." Ähnlich Jone, Katholische Moraltheologie, S. $170 f$. 
Unterschied zwischen „Sterbenlassen“ und „Töten“. (S.187) Sterbenlassen ist von jeher praktiziert worden, weil „überhaupt im Leben nicht in jedem Fall alle denkbaren Mittel angewendet werden, um den Tod hinauszuschieben" (S. 187). Sterbenlassen ist nicht per se ethisch verwerflich: Unter gewissen, nicht näher ausgeführten Bedingungen hält Bonhoeffer das bewußte Sterbenlassen unter Verzicht auf lebensverlängernde Maßnahmen für zulässig, denn die ärztliche Pflicht zum Lebenserhalt endet dort, wo dem Leben Grenzen gesetzt sind. Mit diesem Argument will Bonhoeffer die sinnlose Quälerei schwerkranker sterbender Menschen durch Lebensverlängerung vermieden sehen und damit durchaus Rücksichtnahme gegenüber ihren Bedürfnisse walten lassen, aber gleichzeitig nicht das Mittel der Tötung als Form der Rücksichtnahme zulassen.

Wer die Tötung unschuldiger Kranker mit der "Rücksicht auf die Gesunden“ zu rechtfertigen sucht, macht das Lebensrecht kranker Menschen von ihrem "Nutzwert" für die Gemeinschaft abhängig oder argumentiert wenigstens mit einer unterschiedlichen Bewertung des Lebensrechtes von sozial Wertvollen und sozial Wertlosen. (S.187) Gerade das „sog. lebensunwerte Leben unheilbarer Kranker" hält Bonhoeffer aber für sozial wertvoll, da es - hier nimmt er beinahe wörtlich eine Formulierung seines Vaters auf ${ }^{99}$ - „bei den Gesunden, bei Ärzten Pflegern, Verwandten das höchste Maß sozialer Opferbereitschaft und wahrsten Heldentums auslöst", was ein „höchst realer Nutzwert" für die Gemeinschaft sei. (S.189) Die Unterscheidung zwischen lebenswertem und lebensunwertem Leben zerstört früher oder später das Leben selbst, sie produziert "Willkür“, und es ist zu befürchten, „daß bald diese, bald jene Gruppe von Menschen von dem Vernichtungsurteil getroffen würde“. (S.189) Auch aus theologischer Sicht ist diese Unterscheidung abzulehnen: „Das Recht auf Leben besteht im Seienden und nicht in irgendwelchen Werten. Es gibt vor Gott kein lebensunwertes Leben; denn das Leben selbst ist von Gott wertgehalten." (S.188) Die Frage, ob es sich in Fällen schwerer „Idiotie“ überhaupt um Menschen handle, sei „so naiv, daß sie kaum einer Antwort bedürfe“. (S. 190)

Einen Grenzfall sieht Bonhoeffer dort, wo das Leben des Kranken nicht den materiellen Nutzen, sondern das Leben der Gemeinschaft bedroht. Dieser Grenzfall liegt nicht vor, weil die Versorgung Kranker die Lebenshaltung eines Volkes nicht ernsthaft beeinträchtigt und auch nie nur im entferntesten die Ausgaben für Luxusartikel erreicht. (S.190) Hypothetisch ist der Fall denkbar, daß auf einem Schiff die Pest ausbricht und nach menschlichem Ermessen das Leben der Gesunden nur durch den Tod des Kranken gerettet werden kann. In diesem Fall ist der Konflikt unlösbar, die Entscheidung, ob eine Tötung zulässig ist, muß „offen“ bleiben (S.191), aber auch eine freiwillige Selbsttötung als Opfer ist denkbar - das Beispiel des überfüllten Rettungsbootes - (S. 198). Da diese hypothetischen Grenzsituationen nicht gegeben sind, kommt Bonhoeffer zu dem Schluß, daß die Rücksicht auf die Gesunden die Tötung nicht rechtfertigen

99 Siehe S. $63 \mathrm{f}$. 
kann. Zusammengefaßt sieht er dieses Urteil in Ex. 23,7: „Den Unschuldigen sollst Du nicht erwürgen". (S. 191)

Bonhoeffer konstatiert, daß die „Euthanasie“-Aktion weder soziale noch wirtschaftliche noch hygienische Wurzeln hat, sondern "weltanschauliche“. Diese Weltanschauung charakterisiert er folgendermaßen: „Es soll hier der übermenschliche Versuch gemacht werden, die menschliche Gemeinschaft von sinnlos erscheinender Krankheit zu befreien. Es wird ein Kampf mit dem Schicksal oder, wie wir auch sagen können: mit dem Wesen der gefallenen Welt selbst aufgenommen. Man meint, mit rationalen Mitteln eine neue gesunde Menschheit schaffen zu können. Zugleich hält man Gesundheit für den höchsten Wert, dem alle anderen Werte geopfert werden müßten. Rationalismus und Biologisierung des menschlichen Lebens verbinden sich in diesem vergeblichen Unterfangen, durch das das Recht alles Geschaffenen auf Leben und damit schließlich aller Gemeinschaft überhaupt zerstört wird." (S. 191)

Anknüpfend an seine früheren Überlegungen, derzufolge Krankheit Teil der gefallenen Welt ist und bleibt ${ }^{100}$, kann Bonhoeffer die nationalsozialistische Weltanschauung als den hybriden Versuch kennzeichnen, einen neuen, makellosen und gesunden Menschen zu schaffen. Doch kommt das hierbei errichtete irdische Gesundheitsparadies tatsächlich eher der Hölle nahe: Bei dem Versuch, Krankheit abzuschaffen, wird nicht etwa Gesundheit erreicht, sondern Leben und Gemeinschaft überhaupt zerstört.

\section{Das „Recht auf Fortpflanzung“ im Widerspruch zur Zwangssterilisation}

Auch Bonhoeffers Haltung zur Sterilisation ist durch eine Auseinandersetzung mit katholischen Positionen gekennzeichnet. Für die katholische Position in der Sterilisationsfrage war die in der Enzyklika casti connubii geübte Unterscheidung zwischen erlaubten "natürlichen“ und unerlaubten „künstlichen“ Formen der Empfängnisverhütung maßgeblich ${ }^{101}$. Dabei wurde die Sterilisation, die erzwungene ebenso wie die auf Wunsch, als ein verbotener „künstlicher" Eingriff in die "natürlichen" Zeugungsvorgänge abgelehnt. Erlaubt war dagegen, auch zu eugenischen $Z$ wecken, die Beachtung der unfruchtbaren Zeiten im Zyklus der Frau, die man im Anschluß an den französischen Arzt Pouchet besonders in der dritten Woche nach der Menstruation vermutete ${ }^{102}$, bis sich in den dreißiger Jahren die gegenteilige Auffassung von Knaus/Ogino durchsetzte, derzufolge gerade in diese Zeit der Eisprung und damit die Empfängnis fällt.

\footnotetext{
${ }^{100}$ Siehe S. 129.

101 Vgl. Rundschreiben Pius XI.

102 Empfohlen von Jone, Katholische Moraltheologie, S.617; Lindner, Usus matrimonii, S.219. Bei sorgfältiger Beachtung dieser Methode, so faßt es Ranke-Heinemann sarkastisch zusammen, „bekamen diejenigen Leute Kinder, die keine wollten, und die, die [...] welche wollten, bekamen sie nicht"; dies., Eunuchen, S. 304.
} 
Bonhoeffer lernte die Schattenseiten dieses Argumentationsweges im Kloster Ettal kennen: „Ich sprach lange mit dem Abt und Johannes ${ }^{103}$ darüber. Sie meinten, die Stellung der Kirche zur Empfängnisverhütung sei der Hauptgrund für die meisten Männer, nicht mehr zur Beichte zu kommen. Mir scheint die Beichtpraxis an diesem Punkt auch wirklich höchst gefährlich zu sein. Ohne Reue wird natürlich nicht absolviert, was soll aber eine Reue, deren Unwahrhaftigkeit sich alle drei Tage aufs neue beweist? Das schafft Heuchelei. Faktisch ist die Erkenntnis der Sündhaftigkeit dieses Tuns nicht vorhanden und dann hat alles keinen Sinn. Ich glaube man muß hier viel Freiheit geben "104.

Im Unterschied zur kirchenamtlichen katholischen Position will Bonhoeffer den Zwang und nicht die „Künstlichkeit“ des Eingriffes zum zentralen Punkt seiner Kritik an der nationalsozialistischen Sterilisationspolitik machen. Hierzu sucht er ein „Recht zur Eheschließung“ und ein „Recht zur Fortpflanzung“ zu begründen und entfalten. In dem Recht auf Erhaltung des Lebens ist das Recht auf freie Partnerwahl in persönlicher Entscheidung und damit das Recht auf Fortpflanzung eingeschlossen. (S.199) Freilich ist auch die ungewollt kinderlose Ehe vollgültig und vollständig. Ehe ist mit der Schöpfung der ersten Menschen gegeben. Es dürfen zwar alle Gründe ständischer, wirtschaftlicher, religiöser, konfessioneller, biologischer oder völkischer Art von den Ehepartnern bei der freien Wahl des Ehegatten berücksichtigt werden, aber sie dürfen ihnen nicht von Dritten aufgenötigt werden. (S. 201) Eine Behinderung der freien Partnerwahl sieht Bonhoeffer im Verbot einer Eheschließung zwischen "Juden" und „Deutschblütigen“ („Nürnberger Gesetze“), im „Zwang zur Erzeugung eines bestimmten menschlichen Typs" (vermutlich spielte er auf die SS-Organisation "Lebensborn“105 an) und in der Einschränkung gemischt-konfessioneller Ehen durch das katholische Eherecht ${ }^{106}$. (S. 201) Zur Begründung verweist er auf das Wesen der Ehe: „Ehen werden weder durch die Kirche, noch durch den Staat geschlossen und empfangen auch nicht erst durch diese Institutionen ihr Recht. Die Eheschließung erfolgt vielmehr durch die Ehegatten. Die Tatsache, daß die Ehe öffentlich vor dem Staat und öffentlich vor der Kirche geschlossen wird, bedeutet nichts als die staatliche und kirchliche Anerkennung der Ehe und der ihr innewohnenden Rechte. Das ist lutherische Lehre." (S. 202) - Hier wäre Luthers Schrift Vom ebelichen Leben zu vergleichen, in der Luther die Ehe als ein „leiblich Ding" kennzeichnet und ein sakramentales Eheverständnis ablehnt. Weil Heiden und Ungläubige „Bo wol eyn man und weyb von gott wol und gutt geschaffen als S. Peter und S.Paul und S.Lucia", ist jedweder Kontakt mit ihnen und damit auch die Ehe legitim: „Wie ich nu mag mit eym heyden, Juden,

${ }^{103}$ Prälat Johannes Neuhäusler, kirchenpolitischer Referent Kardinal Faulhabers, Oktober 1940 Bericht über Umfang der "Euthanasie“-Aktion, 4.2. 1941 Verhaftung, bis $1945 \mathrm{KZ}$ Oranienburg und Dachau; vgl. Akten V, S.235-237.

104 GS II, S. 401, Bonhoeffer an Bethge, 20.1. 1941.

105 Vgl. Becker, Rassenhygiene, S.273.

106 Vgl. Dombois, Mischehe. 
Turcken, ketzer essen, trincken, schlaffen, gehen, reytten, kauffen, reden und handeln, alßo mag ich auch mit yhm ehelich werden und bleyben ${ }^{\text {“ }}{ }^{107}$.

Das Recht werdenden Lebens wird nach Bonhoeffers Ansicht bei der Eheschließung von den Eheleuten anerkannt und steht deshalb nicht zu ihrer Verfügung. „Die Tötung der Frucht im Mutterleib [...] ist nichts anderes als Mord.“ (S. 203) Die Frage, ob es sich bereits um menschliches Leben handelt, ist irrelevant, weil sie die Tatsache verkennt, daß „Gott hier jedenfalls einen Menschen schaffen wollte." (S. 203) Lediglich die Tötung der Frucht bei Lebensgefahr für die Schwangere (heute medizinische oder vitale Indikation genannt) erscheint Bonhoeffer zulässig, weil hier, solange die Frucht noch nicht allein lebensfähig ist, das Leben der Mutter gegen das der Frucht allein abgewogen werden muß. (S.204 Anm. 4)

Die Sichtweise mehrerer katholischer Ethikentwürfe, die als „primären Ehezweck“ die Fortpflanzung, als „sekundären“ die Geschlechtsgemeinschaft nennen, kehrt Bonhoeffer um, indem er ein „von dem Recht auf Fortpflanzung unterschiedenes, wenn auch wesenhaft nie gänzlich zu trennendes, in der Liebe der Eheleute zueinander gegründetes Recht auf leibliche Gemeinschaft" anerkennt und zugleich erklärt, „daß dieses Recht der Natur doch mit Vernunft wahrgenommen werden will, eben weil es ein menschliches Recht ist". (S.191f.) Verantwortliche Empfängnisverhütung ist vom Schwangerschaftsabbruch zu unterscheiden. Sexualität hat ihren Wert in sich, ist von der Fortpflanzung zu unterscheiden, aber nicht zu trennen. Eine „konkrete, verantwortliche Empfängnisverhütung “, bei der die Vernunft den technischen Stand der Verhütung bestimmt, ist vertretbar. Der Ansicht vieler Bevölkerungspolitiker und Statistiker, die Deutschen könnten durch den Gebrauch von Empfängnisverhütungsmitteln aussterben ${ }^{108}$, tritt Bonhoeffer mit dem Hinweis darauf entgegen, daß der Rückgang der Geburten in etwa durch den Rückgang der Kindersterblichkeit kompensiert werde. (S. 205)

„Die radikalste Form der Verhütung unerwünschter Nachkommenschaft ist die freiwillige oder durch staatliches Gesetz erzwungene Sterilisation. " (S. 209) Die Schwere und der Ernst dieses Eingriffes dürfen nicht verkannt werden, hat doch der Leib in sich ein Recht auf Unantastbarkeit, welches jedermann gegenüber sich selbst und gegenüber anderen zu respektieren hat. Während die Amputation eines Körpergliedes in gewissen Fällen zur Erhaltung des ganzen Leibes geboten sein kann, dient die Sterilisation nur der "Ermöglichung bestimmter unter einem starken physischen Drang stehender leiblicher Funktionen“. (S.210) Dennoch hält Bonhoeffer die freiwillige Sterilisation unter Vorbehalt für zuläs-

${ }^{107}$ Luther, Werke Bd. 10,2, S. 283 Z. 9-11, 14-16; dazu Scheurl/Sehling, Eherecht, S. $211 \mathrm{f}$.

108 Bonhoeffer nennt keine Namen, vgl. aber z. B. Otto/Stachowitz, Vererbungslehre, S.72. Im Widerspruch dazu behauptete die NS-Propaganda auch, die Deutschen seien ein „Volk ohne Raum“. Tatsächlich wurden „Raum ohne Volk“ und „Volk ohne Raum" von der Propaganda gleichermaBen als Gefahr beschworen. 
sig: Sie komme allerdings "nur bei Krankheitsfällen in Betracht" und nur dort, wo alle anderen Möglichkeiten der Verhütung versagen.

Von der freiwilligen Sterilisation grenzt Bonhoeffer die "staatlich erzwungene“ Sterilisation zwecks Verhütung staatlicherseits unerwünschten Nachwuchses scharf ab. Wenn der Staat auch zu Recht voraussetze, daß Erbkranke nicht enthaltsam leben, so berechtige ihn das nicht zu einem derartig schweren Eingriff in das Recht des einzelnen und die Unantastbarkeit seines Leibes. Außerdem besteht die Gefahr, daß dort, wo diese Grenze einmal überschritten ist, auch alle anderen Grenzen, die „mit der leiblichen Unantastbarkeit gegeben“" sind, „fallen“ (S.210), eine Anspielung auf die „Euthanasie“-Aktion. Als Mittel zur Verhütung unerwünschten Nachwuchses bietet sich außerdem die "Internierung“ an. (S.211)

Die Beantwortung der Frage, ob die Sterilisation „überhaupt die erwünschten [eugenischen] Folgen haben kann" bzw. „in welchen einzelnen Krankheitsfällen sie als berechtigt zu gelten hätte", bezeichnet Bonhoeffer in einer Fußnote als „Sache der medizinischen Wissenschaft“ und fügt hinzu: „Hier ist von der Voraussetzung ausgegangen, daß die Sterilisation in bestimmten Fällen ärztlich sinnvoll und zweckmäßig ist." (S.192 Anm. 12) Offenbar scheint Dietrich Bonhoeffer die These seines Vaters, derzufolge Sterilisation bei rezessiv erblichen Krankheiten nicht das erwünschte Ergebnis erzielen könne und das Erbgesundheitsgesetz in bezug auf die einzelnen Krankheiten unzureichend begründet sei, gekannt zu haben. Zugleich wird aber sein Bestreben deutlich, die theologische Ablehnung der Zwangssterilisation nicht vom empirischen Ergebnis der Naturwissenschaft abhängig zu machen: Das Recht auf Fortpflanzung soll selbst für den Fall gelten, daß man zumindest einige Krankheiten durch Sterilisation erfolgreich bekämpfen kann.

Nachdem Bonhoeffer ein staatlich erzwungenes Sterilisationsgesetz als eine Verletzung der Rechte leiblichen Lebens abgelehnt hat, bleibt die Frage, wie man sich zu verhalten hätte, wenn ein solches Gesetz dennoch positives "Recht" geworden ist. Einem Vorverweis kann entnommen werden, daß Bonhoeffer diesem Problemkreis ein späteres Kapitel widmen wollte, in dem auch ähnliche Fragen wie der Umgang "gemischtrassiger" Brautpaare mit den „Nürnberger Gesetzen“ und gemischtkonfessioneller Brautpaare mit dem katholischen Kirchenrecht behandelt werden sollten. (S.202f.) Die „Einstellung des Kranken und des Arztes", so deutet er in Kürze an, wird bestimmt von der "Gehorsamspflicht gegenüber der Obrigkeit " (S.211), welche Bonhoeffer zufolge immer dort endet, wo die Obrigkeit den Menschen „direkt zum Verstoß gegen das göttliche Gebot zwingt"109.

Bonhoeffer hat das hier angekündigte Kapitel nicht mehr geschrieben. Es kann aber anhand seiner Überlegungen zur Verantwortungsethik vermutet werden, in welche Richtung seine Argumentation verlaufen wäre. Die bloße Ignorierung

${ }^{109}$ So jedenfalls an einer dritten Stelle in Staat und Kirche; vgl. Bonhoeffer, Ethik ${ }^{11}$, S. 364. 
von Gesetzen, welche die Rechte leiblichen Lebens verletzen, dürfte kaum "wirklichkeitsgemäß“ im Sinne Bonhoeffers sein. Gelten z. B. Strafandrohungen wie bei den „Nürnberger Gesetzen“, wird verantwortliches Handeln dem Partner gegenüber das Risiko einer Inhaftierung wegen „Rassenschande“ zu berücksichtigen haben. Steht ein/e Patient/in vor der Frage, ob er oder sie sich in ärztliche Behandlung begeben soll, wird verantwortliches Handeln dem eigenen Körper gegenüber das Risiko einer Anzeige mit möglicher Sterilisationsfolge gegenüber dem Risiko einer Nicht-Behandlung abzuwägen haben. Steht ein Arzt/eine Ärztin vor der Entscheidung, einen Patienten/eine Patientin anzuzeigen, eine Sterilisation durch Gutachten zu fordern oder abzulehnen usw., wird verantwortliches Handeln die Folgen der einen oder anderen Entscheidung für die von ihr betroffenen Menschen zu berücksichtigen haben und immer danach trachten müssen, das unter den gegebenen Umständen Bestmögliche für sie zu erreichen. Dabei wird als Ziel angestrebt, „nicht das ,absolut Gute' zu verwirklichen, welches gerade das Schlechteste sein kann, sondern [...] in Selbstbescheidung das relativ Bessere dem relativ Schlechteren vorziehen". (S. 260) Freilich wird das relativ Bessere nie ohne die Bereitschaft zur Schuldübernahme getan werden können.

\section{Karl und Dietrich Bonhoeffer im Vergleich}

Ein Vergleich zwischen den Positionen und Wirkungsmöglichkeiten eines Mediziners und eines Theologen wird nie von völlig gleichen Bemessungskriterien ausgehen können, denn das Aufgabenfeld beider bleibt unterschiedlich. Bei Karl und Dietrich Bonhoeffer sind Theorie und Praxis unterschiedlich gewichtet, auch wenn der Arzt gleichzeitig Wissenschaftler ist und der theologische Ethiker im konkreten Streit der Bekennenden Kirche involviert war. Die Intention der verantwortlichen Schadensbegrenzung, die der Mediziner praktizierte, ging in die Verantwortungsethik des Sohnes ein; Karl Bonhoeffers Prämisse vom Persönlichkeitsrecht erschien bei Dietrich Bonhoeffer unter den Rechten natürlichen Lebens.

Was das konkrete Handeln gegenüber der "Euthanasie" betrifft, gibt es große Parallelen. Beide haben sich vor 1940 kaum mit der "Euthanasie“ beschäftigt ${ }^{110}$, danach aber im Rahmen ihrer Möglichkeiten zu verschiedenen Protestversuchen beigetragen. Karl Bonhoeffer ermöglichte die Rezension eines Freundes in dem von ihm herausgegebenen "Centralblatt für Neurologie und Psychiatrie“. Dietrich Bonhoeffer engagierte sich in der Bekennenden Kirche für eine Kanzelabkündigung, betrieb selbst die Information des Auslandes, was nach nationalsozialistischer Auffassung einen "Landesverrat" einschloß. Das Interesse beider war durchaus fachübergreifend: Karl Bonhoeffer nahm während der „Euthanasie“ ebenso Kontakte zu den Theologen Wurm, von Bodelschwingh und Braune auf,

${ }^{110} \mathrm{Zu}$ Karl Bonhoeffer siehe S. 102. Von Dietrich Bonhoeffer ist eine Stellungnahme nicht bekannt. 
wie Dietrich Bonhoeffer sich für die Verweigerung einiger Ärzte interessierte, die für die Durchführung der „Euthanasie“-Aktion geforderten Fragebögen auszufüllen ${ }^{111}$. Dietrich Bonhoeffer berichtete dem Vater von der „Euthanasie“-Aktion in den staatlichen Anstalten Pommerns ${ }^{12}$, Karl Bonhoeffer ließ den Sohn an seinen Gewissenskonflikten im Umgang mit dem Erbgesundheitsgesetz teilhaben $^{113}$. In der ethisch theoretischen Verarbeitung kommt der Theologe begreiflicherweise besser zum Zuge: Eine ausführliche Begründung, weshalb die „Euthanasie"-Aktion abzulehnen sei, hat nur Dietrich Bonhoeffer formuliert, während dieser Aspekt bei Karl Bonhoeffer im Verhältnis zu seinem „Rückblick auf das nationalsozialistische Sterilisationsgesetz" zu kurz kam. Indem Dietrich Bonhoeffer die Lebenserhaltung zu den ärztlichen Pflichten rechnet, die von Dritten im äußersten Grenzfall auch gegen den Willen des Patienten oder der Patientin zu wahren ist, schließt er sich der ärztlichen Tradition seines Vaters an, der im äußersten Grenzfall depressive Suizidkandidaten auch gegen ihren Willen in eine geschlossene psychiatrische Anstalt einzuweisen forderte ${ }^{114}$. Er ist sich aber auch bewußt, daß es sich um eine vorübergehende Notlösung für einen problematischen Grenzfall handelt; der Arzt ist dem Leben und dem Willen des Patienten verpflichtet, und nur im äußersten Konfliktfall hat er dem Leben gegenüber dem Willen den Vorrang zu geben. Die Unterscheidung zwischen Töten und Sterbenlassen hat bei Karl Bonhoeffer keine direkte Parallele, entspricht aber, worauf Dietrich Bonhoeffer auch mit Recht hinweist, einer von jeher in der Medizin geübten Unterscheidung.

Dietrich Bonhoeffers Ablehnung der Zwangssterilisation bei vorsichtiger Bejahung eugenisch indizierter Sterilisation auf Wunsch trifft sich im Ergebnis genau mit der Position seines Vaters. Beide haben die Sterilisation als Mittel der Empfängnisverhütung (aus sozialen Gründen, etwa zur Begrenzung der Kinderzahl) nicht ausdrücklich diskutiert, sie mag aber mit der Argumentation beider vereinbar erscheinen. Obwohl Dietrich Bonhoeffer das "Recht auf Fortpflanzung" primär als Abwehrrecht gegenüber dem Staat formuliert, gilt es bei ihm wie bei seinem Vater grundsätzlich auch gegenüber Dritten, insbesondere gesetzlichen Vertretern. Während Karl Bonhoeffer in seinem Gutachten von 1923 das Persönlichkeitsrecht als Voraussetzung für die Behandlung der Zwangssterilisation kurz anführt, ausführlich aber die medizinische Seite, besonders die vererbungswissenschaftliche behandelt, stellt Dietrich Bonhoeffer die von ihm als Rechte natürlichen Lebens formulierten Persönlichkeitsrechte in den Mittelpunkt seiner Argu-

${ }^{111} \mathrm{Vgl.} \mathrm{den}{ }_{\text {"confidential report }}$ " Visser't Hoofts S. 126.

112 Siehe S. 103.

113 So schrieb Karl Bonhoeffer an Dietrich Bonhoeffer am 2.2. 1934: Im letzten Semester sei er "emotionell recht bewegt" und durch die "neuen Gesetze" in vielerlei Hinsicht zu "allerhand neuen Überlegungen " genötigt gewesen; vgl. DBW 13, S. 89 f. Der sehr indirekt gehaltene Charakter dieser Andeutungen erklärt sich dadurch, daß der Brief nach England ging und Briefe ins Ausland von der Gestapo besonders intensiv mitgelesen wurden.

114 Siehe S.65f. 
mentation. Indem der Sohn seine Überlegungen systematisch in den größeren Zusammenhang der Menschenrechte stellt, verleiht er ihnen ein Gewicht, das den sporadischen Äußerungen Karl Bonhoeffers zu den Persönlichkeitsrechten nicht beigemessen werden kann. Dafür hat Dietrich Bonhoeffer allerdings die Vererbungsfrage, der bei der Legitimierung bzw. Delegitimierung des Erbgesundheitsgesetzes ein großes Gewicht zukam, erstaunlich beiläufig behandelt. Eine ausführlichere Berücksichtigung vererbungswissenschaftlicher Argumente wäre auch im Sinne von Dietrich Bonhoeffers Argumentation „sachgemäßer“ gewesen.

Dietrich Bonhoeffer befürwortet - offenbar im Anschluß an den katholischen Moraltheologen Ludwig Ruland (S.211 Anm. 132) - die Internierung Erbkranker. Dabei ist er sich offensichtlich nicht bewußt, daß die Frage, ob dies zwangsweise geschehen darf, ein ethisch gesondert $\mathrm{zu}$ diskutierendes Problem bildet. Der Sache nach widerspricht es sowohl dem „Recht auf Fortpflanzung“ (S.212) wie dem Recht auf „Freiheit des leiblichen Lebens“, welches Schutz vor „willkürlicher Beraubung der Freiheit durch Gefangennahme Wehrloser und Unschuldiger durch [...] Verhaftung" (S.215) einschließt. Im Unterschied dazu erscheinen Karl Bonhoeffers Ausführungen zur Zwangseinweisung, in denen aus gutem Grund die Möglichkeit einer eugenisch indizierten Zwangsasylierung nicht vorgesehen war ${ }^{115}$, in sich konsistenter. Hier brachte der Psychiater, dem das Problem der Zwangseinweisung berufsbedingt sehr viel besser vertraut war, eine größere Sensibilität für die tatsächlich auftretenden Probleme auf.

Ähnliches gilt für die Behandlung des Schwangerschaftsabbruches. Indem Dietrich Bonhoeffer ihn mit „Mord“ gleichsetzt, hält er fast jeden Schwangerschaftsabbruch (ausgenommen vitale Indikation) für grundsätzlich verboten. Zwar diskutiert er nicht ausdrücklich, inwiefern der Staat diesen „Mord“ auch strafrechtlich verfolgen müsse, er scheint dies aber vorauszusetzen. Sein grundsätzliches Verbot des Schwangerschaftsabbruches schließt das von den Nationalsozialisten 1935 erlassene „Gesetz zur Änderung des Gesetzes zur Verhütung erbkranken Nachwuchses“ ein, demzufolge voraussichtlich erbkranker Nachwuchs auf Wunsch der werdenden Mutter nach Genehmigung durch das Erbgesundheitsgericht abgetrieben werden durfte ${ }^{116}$. Im Unterschied dazu hatte Karl Bonhoeffer in der Weimarer Republik den Schwangerschaftsabbruch auch dann für zulässig erklärt und die entsprechende Rechtsprechung begrüßt, wenn nicht das Leben, sondern „nur" die Gesundheit der werdenden Mutter gefährdet war ${ }^{117}$. Es ist dies ein weiteres Beispiel dafür, daß Karl Bonhoeffer durch seine berufliche Nähe zu den bei Schwangerschaftspsychosen auftretenden Problemen auch zu

\footnotetext{
115 Siehe S. $65 f$.

${ }^{116}$ Gesetz zur Änderung des Gesetzes zur Verhütung erbkranken Nachwuchses vom 26.6. 1935, RGBl 1935 I, S.773. Diese Gesetzesänderung gestattete aber keineswegs den Schwangerschaftsabbruch bei „erbgesundem“ Nachwuchs. Im Gegenteil: Das Strafmaß von $\$ 218$ wurde sogar verschärft!

117 Siehe S. $79 f$.
} 
menschlich sensibleren Problemlösungen fähig war. Von seiner pragmatischen Befürwortung des Schwangerschaftsabbruches bei Gesundheitsgefährdung für die Mutter ergibt sich eine Anfrage an Dietrich Bonhoeffers ethisches Urteil, ob man Schwangerschaftsabbruch wirklich mit Mord gleichsetzen kann.

Eine ähnliche Infragestellung ergibt sich bei der rechtlichen Behandlung außerehelicher Sexualität. Karl Bonhoeffer trat dafür ein, Homosexualität unter Erwachsenen strafrechtlich nicht zu verfolgen, und begründete das mit dem für seine pragmatische Argumentationsweise kennzeichnenden Argument, daß Verbote keinen nennenswerten präventiven Effekt hätten ${ }^{118}$. Dietrich Bonhoeffers behandelte im Rahmen des „Rechtes auf Fortpflanzung“ Sexualität überhaupt nur ehelich: Seine gedankliche Prämisse für die Diskussion des Schwangerschaftsabbruches - mit der Eheschließung sei das Lebensrecht werdenden Lebens von den Eheleuten anerkannt - geht an dem Problem vorbei, daß ein nicht unwesentlicher Teil der Abbrüche außereheliche Schwangerschaften betraf. Wir wollen hier nicht die mittlerweile stark gewandelte Auffassung der Sexualität zum Beurteilungskriterium für Ausführungen der dreißiger oder vierziger Jahre erheben. Dennoch hätte im Rahmen von Dietrich Bonhoeffers rechtstheologischen Überlegungen die rechtliche, gerade auch die strafrechtliche Behandlung außerehelicher Sexualität Erwähnung finden können: Die Existenz unehelicher Kinder (außerhalb des Großbürgertums!) war ja kaum zu übersehen.

Während der Psychiater dem Theologen die größere Nähe zu den Problemen seiner ärztlichen Praxis voraus hat, besitzt der Theologe durch seine theologische Prägung eine größere sprachliche Sensibilität. Begriffe wie "minderwertig", „hochwertig“ oder „abartig“, die Karl Bonhoeffer selbst noch nach 1945 unreflektiert als vermeintlich neutrale wissenschaftliche Fachausdrücke benutzt, sind bei Dietrich Bonhoeffer mit Ausnahme eines Barceloner Gemeindevortrages von $1928^{119}$ nicht zu finden: Die christologische These Dietrich Bonhoeffers, daß jedes menschliche Leben von Gott für wert gehalten wird, widerspricht einer menschenverachtenden Terminologie. So verändert die theologische Reflexion auch den Sprachduktus und fördert dessen Humanität. Freilich bedient auch Dietrich Bonhoeffer sich gelegentlich des Ausdrucks "Idiot“.

Trotz aller Unterschiede, bei denen man hier dem Mediziner, dort dem Theologen zustimmen kann, ist für die konkrete ethische Urteilsfindung eine weitgehende Übereinstimmung zwischen den Überlegungen von Vater und Sohn auszumachen. Dies läßt sich auch an wechselseitiger Hochschätzung belegen: Wenn Dietrich Bonhoeffer noch im Gefängnis sein Elternhaus als einen "Schutzwall“ gegen „alle inneren und äußeren Gefahren" charakterisierte, in dem er Zuflucht, Rat, Stille und Klärung suchte, und in seinem eigenen Handeln nicht mehr als ein Bemühen (!) sah, sich überall in dem Geist zu bewähren, den er in seinem El-

\footnotetext{
${ }^{118}$ Vgl. Bonhoeffer, Stellungnahme; zur Homosexualität im zeitgeschichtlichen Kontext vgl. Grau, Verfolgung, S. 93 .

119 Siehe S. 130.
} 
ternhaus vorgefunden habe ${ }^{120}$, dann handelt es sich um Worte, die etwas von einer ungewöhnlich starken Bindung des fast Vierzigjährigen an das Elternhaus und den mächtigen Vater erahnen lassen. Umgekehrt hat sich Karl Bonhoeffer nach Kriegsende sehr für die die Psychiatrie betreffenden Kapitel in der Ethik seines Sohnes interessiert, Eberhard Bethge zur Herausgabe des Fragmentes gedrängt, es Kollegen zur Lektüre empfohlen ${ }^{121}$ und im Nervenarzt durch seinen Schüler Victor von Gebsattel rezensieren lassen ${ }^{122}$.

$120 \mathrm{Vgl}$. Bonhoeffer, Widerstand, S.322f. (Taufpredigt).

121 Vgl. UAT 443/3, NL Binswanger, Korrespondenz Bonhoeffer, Bonhoeffer an Binswanger, 22.11. 1947. Bonhoeffer hatte Binswanger schon die Habilitationsschrift seines Sohnes übersandt; vgl. ebenda, Bonhoeffer an Binswanger, 21.11. 1931. Binswanger bedankte sich, er habe die Schrift "mit großem Interesse gelesen“, sie werde ihm „für die zweite Auflage meiner Psychologie noch gute Dienste tun“; vgl. ebenda. Eine zweite Auflage von Binswangers Psychologie ist allerdings nicht mehr erfolgt.

122 Vgl. Gebsattel, Rez. Dietrich Bonhoeffer, Ethik. 